\title{
Surface photometry of spiral galaxies in NIR: Structural parameters of disks and bulges
}

\author{
C. Möllenhoff and J. Heidt* \\ Landessternwarte, Königstuhl 12, 69117 Heidelberg, Germany \\ Received 29 August 2000 / Accepted 14 November 2000

\begin{abstract}
A sample of 40 bright spiral galaxies $\left(B_{\mathrm{T}}<12\right)$ with low inclination and without bar was observed with $J H K$ filters. For this purpose the MAGIC NICMOS3 camera at the $2.2 \mathrm{~m}$ telescope of the Calar Alto observatory, Spain was used. With a newly developed 2-dimensional algorithm the distribution of the surface brightness was fitted simultaneously by corresponding functions for the disk- and bulge-structure. While the disk was fitted with an exponential function, the bulge profile was fitted with a generalized de Vaucouleurs $R^{\beta}$ law, with the concentration parameter $\beta=1 / n$ as a further fit variable. The correlation of the resulting structural parameters of disks and bulges lead to some interesting relations: 1 . The use of a variable exponent $\beta$ is necessary for a realistic description of the bulge structure; 2. Disks and bulges show similar correlations between effective radius, effective surface brightness and luminosity; 3 . They populate a common strip in the fundamental plane; 4. From the correlations between $\beta$ and Hubble type some conclusions about the formation and evolution of spiral galaxies can be drawn.
\end{abstract}

Key words. galaxies: spiral - galaxies: structure - galaxies: photometry - galaxies: fundamental parameters galaxies: statistics - galaxies: formation

\section{Introduction}

\subsection{The role of surface-brightness profiles for the structure of galaxies}

An important tool for galaxy research is the study of the surface-brightness $(S B)$ distribution (for a comprehensive review see e.g. Simien 1989; Okamura 1988). In most cases the radial structure of the $S B$ distribution is parametrized by simple functions (e.g. exponential) leading to the definition of structural parameters (e.g. central $S B$, scale length). The aim of such studies is a better systematic and quantitative knowledge of the structure of the galaxies and to get keys for a deeper understanding of the evolution and formation of galaxies.

For elliptical galaxies the fitting and study of the $S B$ profiles by a de Vaucouleurs $R^{1 / 4}$ law (de Vaucouleurs 1953) is a well established technique. This method, together with a detailed study of the isophotes and their tiny deviations from pure ellipses were very successful and lead to general new insights into the structure and evolution

\footnotetext{
Send offprint requests to: C. Möllenhoff, e-mail: cmoellen@lsw. uni-heidelberg.de

* Visiting astronomers of the German-Spanish Astronomical Center, Calar Alto, operated by the Max-Planck-Institut für Astronomie, Heidelberg, jointly with the Spanish National Comission for Astronomy.
}

of these objects. (e.g. Bender \& Möllenhoff 1987; Bender et al. 1988, 1989).

Spiral galaxies are more complex since they are built up from a disk, a bulge, and in may cases a bar. The $S B$ profiles of the disks are usually described by an exponential function. The determination of the scale length of the exponential disk has a long tradition (e.g. Freeman 1970; Grosbøl 1985; Courteau 1996). However, the errors in these data are still rather large (Knapen \& van der Kruit 1991), especially if they were obtained from photographic plates. The structure of the bulges is not so clear (e.g. Carollo et al. 1999). In most cases bulges are considered to be similar to elliptical galaxies and their $S B$ profile is described by a de Vaucouleurs $R^{1 / 4}$ function. The $S B$ distribution of bars is not considered in this paper.

\subsection{Fits to the surface-brightness distribution}

There are numerous studies about the decomposition of spiral galaxies in disks and bulges, using a variety of methods. Most analyses of the $S B$ distribution for spiral galaxies were one-dimensional. Either the $S B$ profiles were extracted directly from the images (e.g. Boroson 1981; Kent 1985; Carollo et al. 1998; Bagget et al. 1998), or these profiles were obtained from the fitting of ellipses to the isophotes of the galaxies (e.g. Prieto et al. 1992, Héraudeau \& Simien 1996). The resulting $S B$ profiles were 
then fitted by an exponential function for the disk plus a bulge function (de Vaucouleurs type or exponential). The problem of the ellipse-fitting analyses is that they consider an azimuthal mean of the flux.

In order to separate the non-axisymmetric structures (bars, triaxial bulges, spiral arms) from the axisymmetric components (disk, bulge) two-dimensional fits are necessary (Byun \& Freeman 1995). There exist different approaches for direct surface fits to the two-dimensional $S B$ distribution: sums of Gaussian functions (e.g. Monnet et al. 1992; Emsellem et al. 1994), iterative subtraction of different model components (e.g. Scorza \& Bender 1990, 1995), analysis of 1-dim $S B$ profiles at different position angles (e.g. Andredakis et al. 1995; Peletier \& Balcells 1996), or a nonlinear direct fit of disk- and bulge-models to the $S B$ surface, minimizing a corresponding $\chi^{2}$ (e.g. Byun 1992; de Jong 1996a; Wadadekar et al. 1999).

During the last years it turned out that the classical de Vaucouleurs $R^{1 / 4}$ profile does in general not describe the bulge profile adequately. Exponential profiles were used both for disks and bulges (Andredakis \& Sanders 1994; Broeils \& Courteau 1997; Courteau et al. 1996), or a more general profile $R^{1 / n}, n=1,2,3,4$ was used for the bulges (e.g. Moriondo et al. 1998). A better adaption to different bulge profiles can be made by a fit function with the slope $R^{\beta}$ where the real number $\beta=1 / n$ is a further fit parameter which offers another degree of freedom for the bulge profile (Sersic 1968; Seigar \& James 1998; Andredakis et al. 1995; Wadadekar et al. 1999).

\subsection{NIR surface brightness}

The structural parameters of spiral galaxies, e.g. the exponential scale length of the disk or the effective radius of the bulge, are strongly dependent on color (e.g. Möllenhoff et al. 1999). This is a consequence of the dominating stellar population in the corresponding filter and/or of the effect of dust absorption. For the investigation of the mass distribution of the stars, i.e. the structure of disk and bulge, it is necessary to observe in the near infrared (NIR) wavelength region. The advantage of NIR observations is that they have much less perturbations due to dust or especially bright young stars and that in NIR the distribution of the mass-carrying evolved stars is optimal to observe (e.g. Frogel et al. 1996).

\subsection{Contents of this paper}

The aim of this project is the study of the stellar mass distribution in the disks and bulges of spiral galaxies of different Hubble types. For this purpose we exposed images in $J H K$ of a sample of spiral galaxies of various types. Two-dimensional model functions were fitted to these images by a newly developed fitting algorithm which uses the full 2-dim information of the images and allows to model non-axisymmetric structures. The resulting structural parameters of the different galaxies are correlated, leading
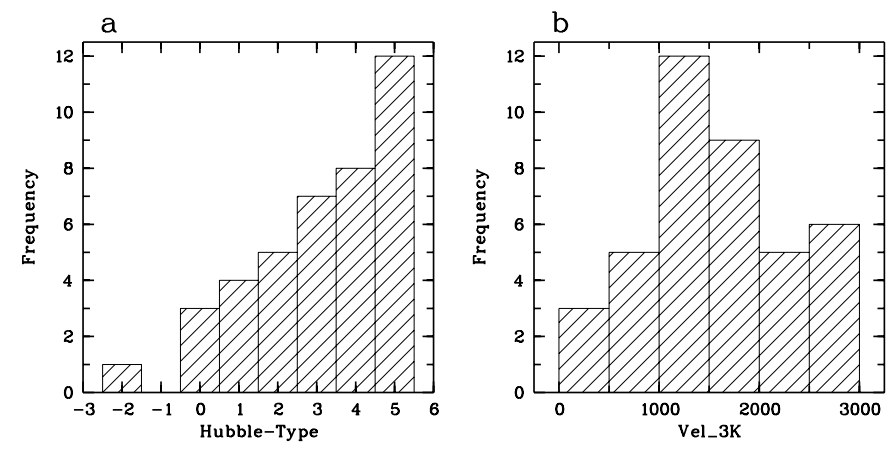

Fig. 1. (a) Distribution of the sample galaxies over the Hubble types. (b) Distribution over radial velocities with regard to the $3 \mathrm{~K}$ background

to some general relations concerning the structure of spiral galaxies. Such relations are useful for a better understanding of the formation and evolution of spiral galaxies. They can give us some clues for the still open question: e.g. were the bulges formed before the disk, or vice versa, or simultaneously?

The paper is organized as follows. In Sect. 2 our sample of spiral galaxies is described, and in Sect. 3 the observations, the data reduction and calibration. In Sect. 4 we present the two-dimensional fit method which fits the twodimensional $S B$ distribution (galaxy image) with a disk and bulge model simultaneously for all pixels. In Sect. 5 we describe results of the fits and give remarks to individual objects. Section 6 presents correlations and statistics of the structural parameters of disks and bulges. In Sect. 7 we discuss the results and Sect. 8 contains the conclusions.

\section{The sample of spiral galaxies}

\subsection{Definition of the sample}

The complete observed sample comprises $\approx 100$ galaxies with $B_{\mathrm{T}}<12$ of Hubble type Sa...Sc and a small number of barred spirals, selected from the Revised ShapleyAmes Catalog (Sandage \& Tammann 1981). In this paper we consider a subsample of 40 galaxies with low inclination and without strong bar. Table 1 lists the observed galaxies, some basic parameters, and our exposure times. The Hubble types were taken from Sandage \& Bedke (1994) and transformed into the T-classes according to RC3 (de Vaucouleurs et al. 1991). $B_{\text {tot }}$ and $v_{3 \mathrm{~K}}$ (radial velocity with respect to the $3 \mathrm{~K}$ background) were taken from RC3. The distance determination is described in the following subsection.

Figure 1a shows the distribution of the sample galaxies over the Hubble types. The late types are predominant due to our selection criterion $B_{\mathrm{T}}<12$, which prefers blue (late type) galaxies. Figure $1 \mathrm{~b}$ shows the distribution of the radial velocities of all galaxies with respect to the $3 \mathrm{~K}$ background. 
Table 1. The observed 40 spiral galaxies with some basic parameters, distances (references explained in the text), and exposure times (in minutes). NGC 5371 was observed twice in two different observational runs

\begin{tabular}{|c|c|c|c|c|c|c|c|c|c|}
\hline No. & NGC & Hubble & & $v_{3 \mathrm{~K}}$ & Dist. & & & $\overline{\exp }$ & \\
\hline & & Type & (mag) & $\left(\mathrm{km} \mathrm{s}^{-1}\right)$ & (Mpc) & Ref. & $J$ & $H$ & $K$ \\
\hline 1 & 278 & 4.0 & 10.70 & 391 & 23.70 & & 9 & 9 & 9 \\
\hline 2 & 628 & 5.0 & 9.79 & 363 & 7.80 & se 5 & 9 & 10 & 9 \\
\hline 3 & 772 & 3.0 & 10.53 & 2188 & 33.46 & & 9 & & 9 \\
\hline 4 & 1023 & -2.0 & 10.09 & 433 & 11.11 & fer & 8 & 9 & 9 \\
\hline 5 & 2196 & 2.0 & 11.39 & 2412 & 43.52 & & 8 & & 8 \\
\hline 6 & 2655 & 0.0 & 10.85 & 1427 & 30.50 & & 9 & 9 & 9 \\
\hline 7 & 2742 & 5.0 & 11.52 & 1404 & 29.61 & & 8 & & 8 \\
\hline 8 & 2775 & 1.0 & 10.48 & 1650 & 21.25 & & 9 & 8 & 9 \\
\hline 9 & 2782 & 1.0 & 12.02 & 2770 & 32.97 & & 9 & 9 & 9 \\
\hline 10 & 2811 & 1.0 & 11.83 & 2821 & 42.12 & & 8 & & 8 \\
\hline 11 & 2841 & 3.0 & 9.60 & 807 & 24.89 & se5 & 9 & 9 & 9 \\
\hline 12 & 2855 & 0.0 & 12.29 & 2224 & 32.88 & & 8 & & 8 \\
\hline 13 & 2964 & 4.0 & 11.64 & 1580 & 27.71 & sos & 8 & & 8 \\
\hline 14 & 2985 & 2.0 & 10.98 & 1384 & 28.00 & & 9 & 9 & 10 \\
\hline 15 & 3147 & 4.0 & 11.25 & 2875 & 48.94 & & 9 & & 9 \\
\hline 16 & 3162 & 4.0 & 11.94 & 1604 & 27.96 & & 8 & & 8 \\
\hline 17 & 3169 & 3.0 & 11.02 & 1575 & 22.52 & & 9 & & 9 \\
\hline 18 & 3198 & 5.0 & 10.22 & 880 & 14.45 & hst & 8 & & 8 \\
\hline 19 & 3338 & 4.0 & 11.15 & 1636 & 26.87 & sos & 9 & 9 & 9 \\
\hline 20 & 3359 & 5.0 & 10.69 & 1140 & 22.63 & sos & 8 & & 8 \\
\hline 21 & 3368 & 2.0 & 9.77 & 1238 & 11.20 & hst & 9 & 9 & 9 \\
\hline 22 & 3430 & 5.0 & 11.70 & 1864 & 33.39 & & 8 & & 8 \\
\hline 23 & 3626 & 0.0 & 11.75 & 1821 & 34.92 & & 8 & & 8 \\
\hline 24 & 3675 & 3.0 & 10.55 & 1000 & 20.27 & sos & 9 & 9 & 9 \\
\hline 25 & 3705 & 3.0 & 11.18 & 1365 & 21.97 & & 8 & & 8 \\
\hline 26 & 3726 & 5.0 & 10.76 & 1066 & 19.67 & & 8 & & 8 \\
\hline 27 & 3810 & 5.0 & 10.95 & 1335 & 21.20 & & 9 & 9 & 9 \\
\hline 28 & 3898 & 1.0 & 11.32 & 1342 & 37.49 & sos & 9 & & 9 \\
\hline 29 & 4051 & 4.0 & 10.77 & 945 & 21.31 & & 9 & & 9 \\
\hline 30 & 4254 & 5.0 & 10.08 & 2732 & 20.70 & vir & 9 & 9 & 8 \\
\hline 31 & 4303 & 5.0 & 10.27 & 1909 & 20.70 & vir & 8 & & 8 \\
\hline 32 & 4414 & 5.0 & 10.71 & 992 & 19.14 & hst & 8 & & 8 \\
\hline 33 & 4450 & 2.0 & 10.70 & 2272 & 20.70 & vir & 9 & & 9 \\
\hline 34 & 4501 & 4.0 & 9.86 & 2589 & 20.70 & vir & 9 & 9 & 9 \\
\hline 35 & 4535 & 5.0 & 10.33 & 2287 & 16.60 & hst & 8 & & 8 \\
\hline 36 & 4725 & 3.0 & 9.74 & 1487 & 13.00 & hst & 9 & 9 & 9 \\
\hline 37 & 4826 & 2.0 & 8.64 & 700 & 7.63 & & 9 & 9 & 9 \\
\hline 38 & 5248 & 4.0 & 10.63 & 1437 & 22.71 & & 9 & & 15 \\
\hline 39 & 5364 & 5.0 & 10.81 & 1512 & 25.03 & & 9 & & 8 \\
\hline 40 & 5371 & 3.0 & 11.18 & 2740 & 45.24 & & 8 & & 8 \\
\hline 41 & 5371 & 3.0 & 11.18 & 2740 & 45.24 & & 8 & & 9 \\
\hline
\end{tabular}

\subsection{Distance determination}

The determination of the distances is crucial to obtain reliable scale lengths in kpc from the fit results in arcsec. We used a Hubble constant $H_{0}=60 \mathrm{~km} \mathrm{~s}^{-1} / \mathrm{Mpc}$ which is an appropriate mean between two recent determinations (Gibson et al. 2000; Federspiel et al. 1998).

The galaxy distances were calculated from the radial velocities by Tully (1988). These values were compared with the distances of Bottinelli et al. (1984, 1985a), obtained from $21-\mathrm{cm}$ line-widths via a revised $B$-band TullyFisher relation. The distances from Tully and Bottinelli et al. are remarkably similar, so we used their mean values for all those galaxies which are not marked in the column "Ref dist" in Table 1. The marked distance values stem from:

hst: These are calibrator galaxies with Cepheid distances from the HST key project for the determination of $H_{0}$. (e.g. Turner et al. 1998; Gibson et al. 2000; Sakai et al. 2000).

fer: Ferrarese et al. (2000) determined the distances to a number of galaxies, using the HST Cepheid calibrators. vir: Using the Cepheid calibrator galaxies from the HST key project Federspiel et al. (1998) determined the distance to the Virgo cluster to $20.7 \pm 2.4 \mathrm{Mpc}$.

sos: Bottinelli et al. (1985b) improved the distances for 197 galaxies by the "sosie" method. We used these improved values for the mean with Tully.

se5: SIMBAD was searched for dedicated papers on the distance of each galaxy, see Sect. 5 .

\section{Observations and data reduction}

The observations were performed during Jan. 26-30, 1994 and Feb. 16-21, 1995 at the $2.2 \mathrm{~m}$ telescope of the GermanSpanish observatory on Calar Alto, Spain. We used the NIR-camera MAGIC with a NICMOS3 chip of $256 \times$ 256 pixels. The camera was used in high-resolution mode $(0.67 \mathrm{arcsec} / \mathrm{pixel})$ yielding a field of view of $\sim 3 \times 3 \mathrm{arcmin}$ (Herbst et al. 1993). The weather was photometric during most of the nights, the typical seeing was 1.5 to 2.5 arcsec FWHM.

The typical exposure times were 8-9 min on each object in $J, H$ and $K$ filters. Due to the strong and highly variable background, the observations on target were splitted into integrations of $3 \times 10 \mathrm{~s}$ each. In order to remove the bad pixels the telescope was displaced by a few arcsec between each set of $3 \times 10 \mathrm{~s}$ exposure time. Since the sizes of the galaxies observed are typically on the order of the field of view of the MAGIC camera, it was necessary to obtain intermediate sky images with the same duration $(3 \times 10 \mathrm{~s})$. For that purpose, the telescope was offset by 25 arcmin in various directions. The full procedure was controlled by a macro program. During each night several standard stars taken from Elias et al. (1982) were observed in order to set the zero points.

The individual images were sky-subtracted and divided by a dome flat. The most critical part was the subtraction of the sky background, because it can vary spatially and temporarily. Moreover, due to the large size of the galaxies observed, the sky level cannot be determined on the galaxy images directly. Therefore the following strategy for the sky subtraction was adopted: first we constructed a master sky frame by median filtering the full set of sky frames, which were observed right before or after each galaxy exposure. The level of the sky on each individual galaxy frame was estimated by the mean of the sky levels of the two adjacent sky frames. Then the master sky frame was scaled to this estimated sky level and subtracted. Finally the sky-subtracted galaxy images were 
divided by a dome flat, which was constructed from exposures of a blank part of the dome with internal lamps on/off.

The resulting 48 sky-subtracted and flat-fielded images were centred and summed up. A Kappa-Sigma summation procedure was used which removes all bad pixels and cosmic ray hits. It works similar as a vertical median filter procedure, however, without having the problems of flux conservation and enhanced noise of the median filter. The Kappa-Sigma summation has the same high signalto-noise ratio as the simple sum (or mean) of the images.

\section{Two-dimensional fits to the surface-brightness distribution}

\subsection{Equations for the flux distribution}

A two-dimensional surface-brightness function for disk and bulge was fitted simultaneously to the observed flux distribution. For the radial flux distribution of the inclined disk we assumed the exponential law

$F_{\mathrm{d}}(R)=I_{\mathrm{d}} \exp \left(-R / R_{\mathrm{d}}\right)$

where $I_{\mathrm{d}}$ is the central flux density and $R_{\mathrm{d}}$ the radial scale length. No outer truncation radius was used. The disk was assumed to be axisymmetric. This corresponds to the projected elliptical geometry

$R^{2}=\left(x \cos \Phi_{\mathrm{d}}+y \sin \Phi_{\mathrm{d}}\right)^{2} /{Q_{\mathrm{d}}}^{2}+\left(y \cos \Phi_{\mathrm{d}}-x \sin \Phi_{\mathrm{d}}\right)^{2}(2)$

where $\Phi_{\mathrm{d}}$ is the position angle $(\mathrm{PA})$ of the major axis measured counterclockwise from north, and $Q_{\mathrm{d}}=b / a$ is the axis ratio. Thus we have 4 free fit parameters for the disk: $I_{\mathrm{d}}, R_{\mathrm{d}}, \Phi_{\mathrm{d}}, Q_{\mathrm{d}}$

For the flux distribution of the bulge we assumed the generalized exponential radial density law of Sérsic (1968)

$F_{\mathrm{b}}(r)=I_{\mathrm{s}} \exp \left(-\left(R / R_{\mathrm{s}}\right)^{\beta}\right)$

where $I_{\mathrm{s}}$ is the central flux density, $R_{\mathrm{s}}$ is a radial scale length, and the real number exponent $\beta=1 / n$ determines the slope of the projected bulge $S B$ distribution. $\beta$ was treated as another free fit parameter. We used Eq. (3) written in the form of a generalized de Vaucouleurs profile

$F_{\mathrm{b}}(r)=I_{\mathrm{b}} \exp \left(-b_{\mathrm{e}}\left[\left(R / R_{\mathrm{b}}\right)^{\beta}-1\right]\right)$

with

$b_{\mathrm{e}}=1.9986 / \beta-0.327 \approx 2 n-0.33$.

Now $R_{\mathrm{b}}$ is the half-light or effective radius and $I_{\mathrm{b}}$ is the flux density at $R_{\mathrm{b}}$ (half-light or effective surface brightness; Caon et al. 1993). $\beta=1 / n=0.25$ corresponds to the classical de Vaucouleurs profile, $\beta=1$ to a simple exponential profile. The Sérsic parameters $I_{\mathrm{s}}, R_{\mathrm{s}}$ and the generalized de Vaucouleurs parameters $I_{\mathrm{b}}, R_{\mathrm{b}}$ are connected via

$I_{\mathrm{b}}=I_{\mathrm{s}} \exp \left(-b_{\mathrm{e}}\right), \quad R_{\mathrm{b}}=R_{\mathrm{s}} b_{\mathrm{e}}^{n}$.
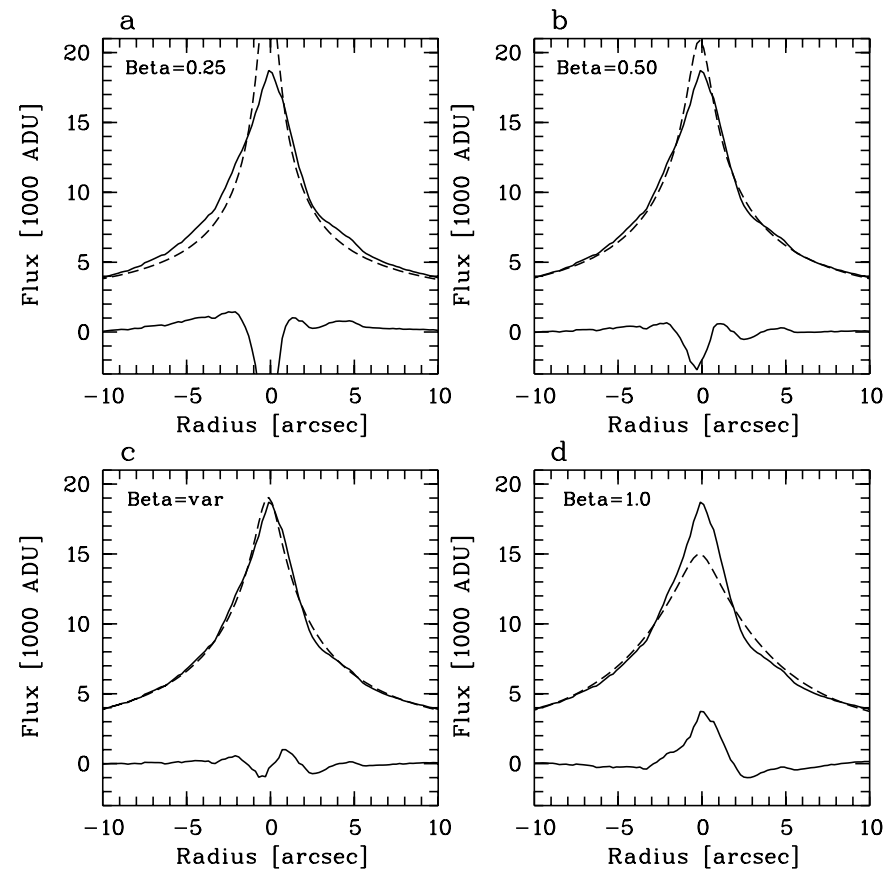

Fig. 2. (a-d) Observed $S B$ profiles (solid lines) of the central bulge region of NGC 1232 ( $V$ band), together with different model fits (dashed lines) and the corresponding residuum (solid lin at bottom) from Möllenhoff et al. (1999). The bottom left diagram (c) shows the best result with $\beta$ as a free fit parameter, leading to $\beta=0.613$. The other diagrams (a), (b), (d) show the unsatisfying fit results with a fixed $\beta=0.25,0.5,1.0$, respectively

Using a similar expression as Eq. (2) for the radius $R$, we obtain the position angle $\Phi_{\mathrm{b}}$ and the ellipticity $Q_{\mathrm{b}}$ as further free fit parameters for the bulge. Thus we have 5 free fit parameters for the bulge: $I_{\mathrm{b}}, R_{\mathrm{b}}, \beta, \Phi_{\mathrm{d}}, Q_{\mathrm{d}}$.

Fitting the total flux $F_{\text {fit }}=F_{\mathrm{d}}+F_{\mathrm{b}}$ simultaneously to the observed two-dimensional $S B$ distribution results in a nonlinear system of equations for the $4+5=9$ free parameters. This system was solved with a LevenbergMarquardt algorithm (e.g. Bevington 1969; Press et al. 1989).

\subsection{The variable bulge exponent $\beta=1 / n$}

The importance of using a variable $\beta=1 / n$ is demonstrated in Fig. 2, where horizontal cuts through the central region of the spiral galaxy NGC 1232 are shown. These plots stems from our 2-dim fits to the $V$ images of this galaxy (Möllenhoff et al. 1999), obtained at the ESO VLT Antu and the FORS focal reducer. These images have a very good spatial sampling $\left(0.2^{\prime \prime} /\right.$ pixel $)$ and signal-tonoise ratio. Therefore they show the bulge profiles with very good resolution and are well suited to demonstrate the strong influence of the exponent $\beta$ on the fit result. Each diagram shows the observed surface-brightness profile as a solid line, the fit model as dashed line, and at bottom the difference (residuum). The bottom left diagram shows the best fit with $\beta$ as a free fit parameter, 
Table 2. Results of a 2-dim disk-bulge fit to a deep $V$ image of NGC 1232. The bulge exponent $\beta$ was chosen as a fixed parameter of different values or was treated as the 9th free fitted structural parameter. The units of $I_{\mathrm{d}}$ and $I_{\mathrm{b}}$ are the digital flux units (ADU) from the CCD

\begin{tabular}{|cl|rc|rr|r|r|}
\hline $\begin{array}{c}\text { Fit } \\
\text { Type }\end{array}$ & $\beta$ & $\begin{array}{c}I_{\mathrm{d}} \\
\text { ADU }\end{array}$ & $\begin{array}{c}R_{\mathrm{d}} \\
\operatorname{arcsec}\end{array}$ & $\begin{array}{c}I_{\mathrm{b}} \\
\text { ADU }\end{array}$ & $\begin{array}{c}R_{\mathrm{b}} \\
\operatorname{arcsec}\end{array}$ & $\begin{array}{c}\text { Bu./Disk } \\
\text { Ratio }\end{array}$ & $\chi^{2}$ \\
\hline fixed & 0.25 & 1338 & 65.3 & 93 & 68.2 & 0.191 & 10025 \\
fixed & 0.50 & 1671 & 62.1 & 928 & 13.4 & 0.046 & 9310 \\
variable & 0.613 & 1739 & 60.9 & 1366 & 10.5 & 0.038 & 9234 \\
fixed & 0.70 & 1796 & 59.9 & 1852 & 8.8 & 0.033 & 9314 \\
fixed & 1.00 & 1869 & 58.5 & 2602 & 7.2 & 0.027 & 9671 \\
\hline
\end{tabular}

leading to a nearly perfect fit with $\beta=0.613$ in this case. The other diagrams show the unsatisfying fit results with fixed $\beta=0.25,0.5,1.0$, respectively. The different $\beta$-values lead to dramatic different bulge models. This is demonstrated in Table 2 . The centre row shows the optimal values with $\beta$ as a free-fitted parameter. A non-optimal $\beta$ does not only influence the bulge parameters very strongly but also the disk parameters, however much less. The last column of Table 2 shows the corresponding behaviour of $\chi^{2}$ (not normalized) from the Levenberg-Marquardt algorithm. The minimum of the $\chi^{2}$ distribution is fairly shallow since $\chi^{2}$ is dominated by the non-axisymmetric residuals in the galaxy which are not fitted, e.g. the spiral arms.

$\beta$ is a concentration parameter for the mass distribution of the bulges. All functions $\exp \left[\left(-R / R_{\mathrm{b}}\right)^{\beta}\right]$ are equal to 1 at $R=R_{\mathrm{b}}$. For large exponents (e.g. $\beta=1$ ) the profile is flatter towards the centre and steeper outside $R_{\mathrm{b}}$ compared to the profile of small exponents (e.g. $\beta=0.25$ ). This means profiles with small $\beta$ are more concentrated in the centre (Fig. 2).

\subsection{PSF and seeing}

The atmospheric turbulence (seeing) deforms the $S B$ profile of the galaxies, especially in the central region where the slope is steep. Therefore the seeing has a big influence on the bulge profile. The two-dimensional point spread function (PSF) was determined from stars and approximated by a two-dimensional Gaussian function. This PSF function was convolved with the model function in each iteration step. The convolved $S B$ fit model was then compared with the observed $S B$ distribution and the control parameter $\chi^{2}$ was computed. If not enough stars for the determination of a PSF were present in the image, the PSF was approximated by a Gaussian profile, with the full width half maximum (FWHM) of the Gaussian profile considered as 10th independent fit variable.

\subsection{Technical procedure of the fits}

On each image the foreground stars were masked, the value of the sky level and the centre of the galaxy were determined. The two-dimensional fit procedure uses a
Levenberg-Marquardt algorithm (e.g. Bevington 1969) for finding an optimal solution to the corresponding system of equations. An important point here is the choice of an adequate weight function, which is normally given by the variance $\sigma^{2}(x, y)$ of the observed $S B$. It has to be considered that for each radius zone the number of pixels is increasing, however with decreasing signal/noise ratio. After different tests a constant weight function turned out to be most suitable.

The iteration procedure was started with a rough guess for the photometric parameters. To ensure that the iteration does not just stop on a side-minimum of the corresponding $\chi^{2}$-surface, it is useful to proceed stepwise. In a first step the fit was performed in a double iteration: the disk parameters were fitted with Levenberg-Marquardt while the bulge parameters were kept fixed. In the next iteration the new disk parameters were kept fixed while now the bulge parameters were iterated, and so on. Then in the second step the parameters from the first step were used as starting values for a simultaneous fit of disk and bulge, which now converged very quickly. A convergent model was reached when $\chi^{2}$ had a minimum and when the relative change of every variable between the iterations was less than $10^{-4}$.

In order to check the quality of the obtained fit result it is important to study the 2-dim residuals. Figures 3-6 shows the observed $J$-images $\left(F_{\text {obs }}\right)$ together with the residuum images $F_{\text {res }}=F_{\text {obs }}-F_{\text {fit }}$ for the spiral galaxies \#1 to \#10 of our sample. The corresponding images of the galaxies \#11 to \#40 (Figs. 4 to 6 ) can be found in the on-line version of this paper. These residuum images give a good impression how good the surface-brightness distribution of each galaxy could be described by our fit model. They show all those structures which could not be described by the adopted elliptical geometry for disk and bulge: asymmetries in the inner disk region, spiral arms, dust lanes, etc.

It is an advantage of this 2-dim $S B$ fit method that the 2-dim residuals allow to judge the results directly. In Sect. 5.2 we give individual remarks to the fit results and the residuals for each galaxy. When comparing the images in Fig. 3 (Figs. 4 to 6 in on-line version) one should keep in mind, that the cuts for the $J$-images are generally between $0,10^{4}$ and $0,10^{5}$, while the cuts of the residuum images are around $-1000,1000$; the the peak-to-peak variations of the residuum images is only a few percents of that of the galaxy images.

Figures 3-6 gives a good qualitative impression of the fit residuals. However, it is furthermore important to study the results quantitatively as well. For each galaxy fit diagrams like Fig. 2 were studied to support.

\subsection{Error estimates}

The fit procedure and its stability were tested extensively with artificial galaxies, including photon noise and seeing convolution. The statistical errors from the 

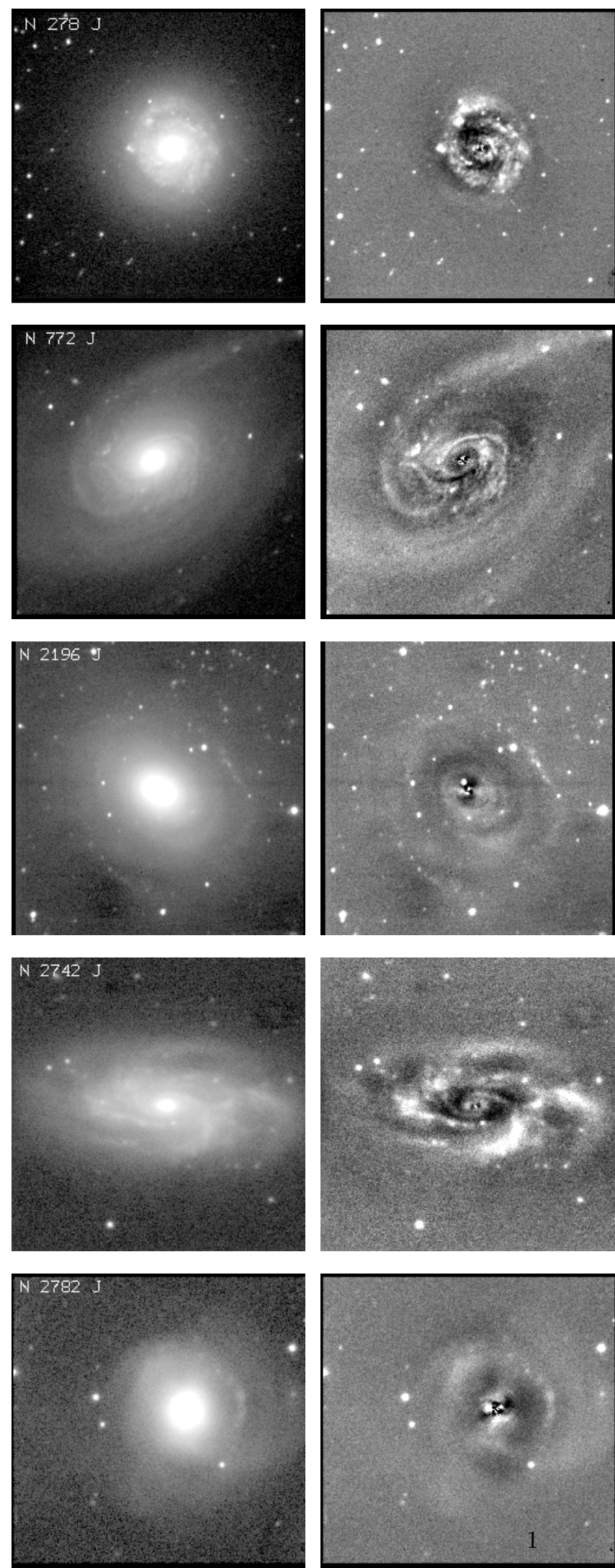
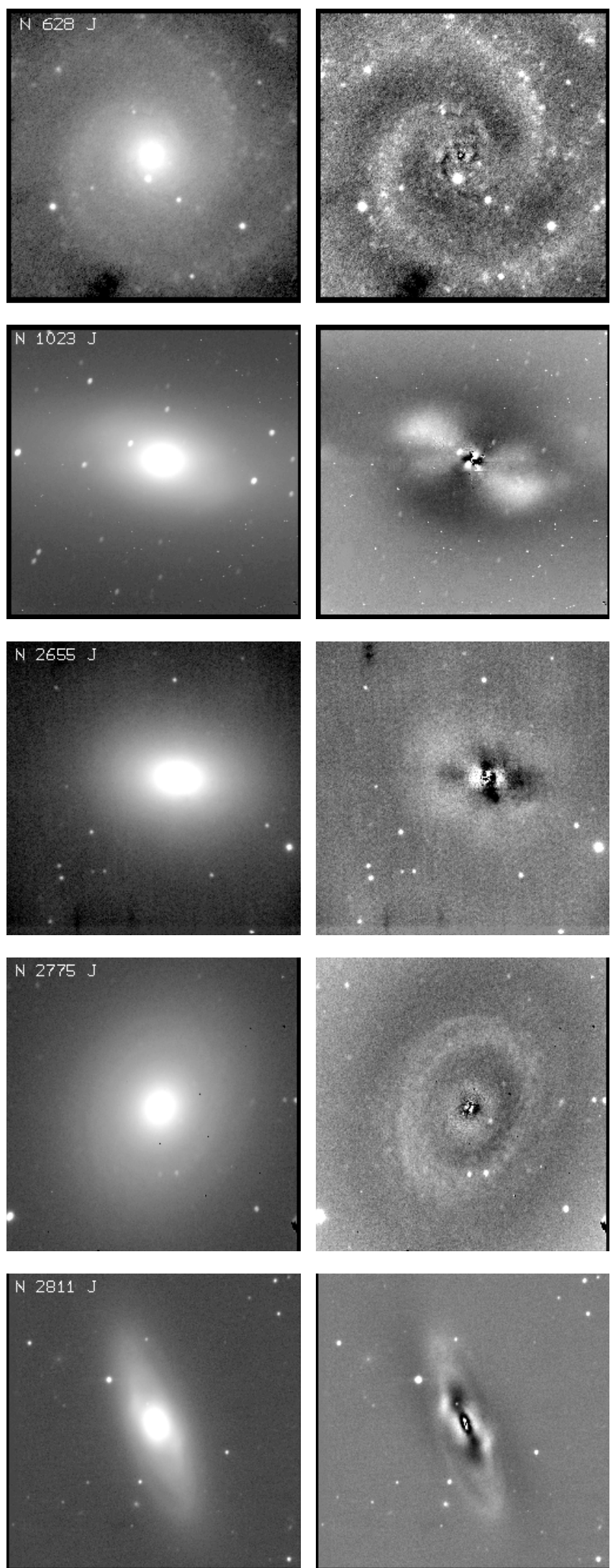

Fig. 3. $J$-filter images and residua of the galaxies \#1 to \#10 of our sample: NGC 278, NGC 628, NGC 772, NGC 1023, NGC 2196, NGC 2655, NGC 2742, NGC 2775, NGC 2782, NGC 2811. The residua were obtained after subtraction of a two-dimensional bulgedisk model from the images. The dynamical range of the residuum images is only a few percents of that of the galaxy images. The size is $\sim 3 \times 3$ arcmin. North is up, east to the left. The corresponding images of the galaxies \#11 to \#40 (Figs. 4 to 6 ) can be found in the on-line version 
Table 3. Structural parameters of the disks. $S B_{\mathrm{c}}$ is the unconvolved central surface brightness in mag/square-arcsec, $R_{\mathrm{d}}$ is the radial scale length of the exponential disk, given in arcsec and in kpc. $\mathrm{PA}_{\mathrm{d}}$ is the position angle of the major axis (line of nodes), and $Q_{\mathrm{d}}=b / a$ is the axis ratio of the model fit to the disk. Each value is given for the corresponding filters $J H K$

\begin{tabular}{|c|c|c|c|c|c|c|c|c|c|c|c|c|c|c|c|c|}
\hline \multirow{2}{*}{\multicolumn{2}{|c|}{ No. NGC }} & \multirow{2}{*}{\multicolumn{3}{|c|}{$\begin{array}{|ccc|}S B_{\mathrm{c}} & (\mathrm{mag} & / \mathrm{sas}) \\
J & H & K\end{array}$}} & \multicolumn{3}{|c|}{$R_{\mathrm{d}}(\operatorname{arcsec})$} & \multicolumn{3}{|c|}{$R_{\mathrm{d}}(\mathrm{kpc})$} & \multicolumn{3}{|c|}{$\mathrm{PA}_{\mathrm{d}} \quad(\mathrm{deg})$} & \multicolumn{3}{|c|}{$\overline{Q_{\mathrm{d}}}$} \\
\hline & & & & & $J$ & $H$ & $K$ & $J$ & $H$ & $K$ & $J$ & $H$ & $K$ & $J$ & $H$ & $K$ \\
\hline 1 & 278 & 16.49 & 15.86 & 16.05 & 14.95 & 15.64 & 16.60 & 1.72 & 1.80 & 1.91 & 37.48 & 42.93 & 47.18 & 0.97 & 0.95 & 0.97 \\
\hline 2 & 628 & 18.54 & 17.82 & 18.30 & 55.70 & 45.38 & 73.08 & 2.11 & 1.72 & 2.76 & 66.30 & 67.08 & 88.76 & 0.95 & 0.91 & 0.73 \\
\hline 3 & 772 & 18.02 & & 16.93 & 30.41 & & 27.94 & 4.93 & & 4.53 & 124.28 & & 121.15 & 0.74 & & 0.79 \\
\hline 4 & 1023 & 17.77 & 16.76 & 16.71 & 121.66 & 93.01 & 82.05 & 6.55 & 5.01 & 4.42 & 79.15 & 80.42 & 78.84 & 0.32 & 0.31 & 0.26 \\
\hline 5 & 2196 & 18.21 & & 18.35 & 23.21 & & 50.15 & 4.90 & & 10.58 & 54.08 & & 46.51 & 0.75 & & 0.31 \\
\hline 6 & 2655 & 17.27 & 16.43 & 16.90 & 13.58 & 12.55 & 16.05 & 2.01 & 1.86 & 2.37 & 83.97 & 84.07 & 76.51 & 0.39 & 0.43 & 0.35 \\
\hline 7 & 2742 & 18.09 & & 17.09 & 33.14 & & 32.91 & 4.76 & & 4.72 & 84.90 & & 84.64 & 0.46 & & 0.45 \\
\hline 8 & 2775 & 18.03 & 17.19 & 17.11 & 62.55 & 47.73 & 44.25 & 6.44 & 4.92 & 4.56 & 164.94 & 155.49 & 158.48 & 0.76 & 0.78 & 0.81 \\
\hline 9 & 2782 & 17.87 & 17.30 & 17.50 & 15.63 & 16.55 & 20.30 & 2.50 & 2.64 & 3.24 & 18.17 & 15.60 & 22.37 & 0.71 & 0.77 & 0.77 \\
\hline 10 & 2811 & 16.98 & & 16.06 & 21.49 & & 21.00 & 4.39 & & 4.29 & 23.93 & & 23.91 & 0.39 & & 0.39 \\
\hline 11 & 2841 & 17.68 & 17.13 & 16.92 & 105.42 & 108.63 & 112.40 & 12.72 & 13.11 & 13.56 & 150.84 & 150.32 & 149.61 & 0.32 & 0.30 & 0.30 \\
\hline 12 & 2855 & 18.71 & & 17.67 & 29.07 & & 21.62 & 4.63 & & 3.45 & 129.09 & & 128.39 & 0.74 & & 0.63 \\
\hline 13 & 2964 & 17.22 & & 16.17 & 18.04 & & 17.30 & 2.42 & & 2.32 & 100.61 & & 103.87 & 0.77 & & 0.78 \\
\hline 14 & 2985 & 18.05 & 17.27 & 17.32 & 30.63 & 26.10 & 31.08 & 4.16 & 3.54 & 4.22 & 2.02 & 174.51 & 161.10 & 0.84 & 0.86 & 0.76 \\
\hline 15 & 3147 & 17.28 & & 16.40 & 23.37 & & 21.60 & 5.54 & & 5.12 & 150.56 & & 150.00 & 0.84 & & 0.82 \\
\hline 16 & 3162 & 18.27 & & 17.27 & 19.21 & & 18.28 & 2.60 & & 2.48 & 10.24 & & 8.24 & 0.79 & & 0.78 \\
\hline 17 & 3169 & 17.81 & & 17.19 & 31.83 & & 36.10 & 3.48 & & 3.94 & 51.57 & & 52.13 & 0.65 & & 0.55 \\
\hline 18 & 3198 & 18.35 & & 17.50 & 82.04 & & 75.09 & 5.75 & & 5.26 & 39.06 & & 39.23 & 0.26 & & 0.26 \\
\hline 19 & 3338 & 17.98 & 17.32 & 17.24 & 26.83 & 29.47 & 25.85 & 3.49 & 3.84 & 3.37 & 94.92 & 94.78 & 91.89 & 0.52 & 0.56 & 0.43 \\
\hline 20 & 3359 & 19.24 & & 18.46 & 63.34 & & 77.71 & 6.95 & & 8.53 & 170.23 & & 169.31 & 0.63 & & 0.64 \\
\hline 21 & 3368 & 16.66 & 15.89 & 16.05 & 35.23 & 2.44 & 37.90 & 1.78 & 1.64 & 1.91 & 133.86 & 34 & 132.19 & 0.66 & 0.67 & 0.64 \\
\hline 22 & 3430 & 18.04 & & 16.80 & 22.23 & & 16.91 & 3.60 & & 2.74 & 32.70 & & 29.48 & 0.54 & & 0.58 \\
\hline 23 & 3626 & 17.00 & & 15.99 & 20.08 & & 18.18 & 3.40 & & 3.08 & 166.93 & & 166.41 & 0.58 & & 0.57 \\
\hline 24 & 3675 & 17.12 & 16.35 & 16.22 & 40.56 & 40.43 & 41.49 & 3.99 & 3.97 & 4.08 & 0.96 & 0.41 & 0.30 & 0.43 & 0.42 & 0.40 \\
\hline 25 & 3705 & 17.42 & & 16.47 & 29.16 & & 26.62 & 3.11 & & 2.84 & 119.36 & & 119.53 & 0.45 & & 0.48 \\
\hline 26 & 3726 & 18.46 & & 17.61 & 61.89 & & 58.32 & 5.90 & & 5.56 & 13.32 & & 12.10 & 0.48 & & 0.46 \\
\hline 27 & 3810 & 17.35 & 16.08 & 15.90 & 18.01 & 16.76 & 15.68 & 1.85 & 1.72 & 1.61 & 23.76 & 21.74 & 23.81 & 0.75 & 0.75 & 0.73 \\
\hline 28 & 3898 & 19.04 & & 18.36 & 56.86 & & 49.47 & 10.33 & & 8.99 & 106.61 & & 102.92 & 0.45 & & 0.27 \\
\hline 29 & 4051 & 18.13 & & 17.26 & 47.75 & & 48.61 & 4.93 & & 5.02 & 128.24 & & 127.70 & 0.42 & & 0.42 \\
\hline 30 & 4254 & 18.23 & 17.47 & 17.24 & 31.30 & 30.13 & 27.31 & 3.14 & 3.02 & 2.74 & 59.63 & 72.96 & 73.80 & 0.92 & 0.90 & 0.87 \\
\hline 31 & 4303 & 17.43 & & 16.50 & 42.84 & & 40.60 & 4.30 & & 4.07 & 12.21 & & 12.37 & 0.62 & & 0.58 \\
\hline 32 & 4414 & 16.11 & & 14.84 & 21.76 & & 19.45 & 2.02 & & 1.80 & 158.30 & & 158.38 & 0.57 & & 0.57 \\
\hline 33 & 4450 & 17.29 & & 16.51 & 34.33 & & 38.31 & 3.45 & & 3.84 & 3.75 & & 2.54 & 0.52 & & 0.52 \\
\hline 34 & 4501 & 16.80 & 15.92 & 15.84 & 45.53 & 42.32 & 45.89 & 4.57 & 4.25 & 4.61 & 140.62 & 140.42 & 139.38 & 0.49 & 0.50 & 0.49 \\
\hline 35 & 4535 & 18.81 & & 17.77 & 68.07 & & 60.01 & 5.48 & & 4.83 & 27.06 & & 28.80 & 0.75 & & 0.77 \\
\hline 36 & 4725 & 17.78 & 17.11 & 17.01 & 49.99 & 50.28 & 54.66 & 3.15 & 3.17 & 3.44 & 44.74 & 44.96 & 44.71 & 0.46 & 0.45 & 0.44 \\
\hline 37 & 4826 & 16.07 & 15.29 & 15.07 & 38.24 & 37.07 & 34.79 & 1.41 & 1.37 & 1.29 & 110.52 & 110.68 & 110.08 & 0.63 & 0.63 & 0.62 \\
\hline 38 & 5248 & 18.81 & & 18.32 & 123.68 & & 194.09 & 13.62 & & 21.37 & 128.68 & & 123.51 & 0.34 & & 0.22 \\
\hline 39 & 5364 & 18.16 & & 17.36 & 36.23 & & 42.28 & 4.40 & & 5.13 & 41.56 & & 38.73 & 0.67 & & 0.70 \\
\hline 40 & 5371 & 18.12 & & 17.22 & 44.17 & & 42.05 & 9.69 & & 9.22 & 21.21 & & 19.18 & 0.71 & & 0.71 \\
\hline 41 & 5371 & 18.17 & & 17.60 & 40.21 & & 51.38 & 8.82 & & 11.27 & 18.21 & & 22.35 & 0.72 & & 0.66 \\
\hline
\end{tabular}

Fig. 4. $J$-filter images and the residua of the galaxies \#11 to \#20 of our sample: NGC 2841, NGC 2855, NGC 2964, NGC 2985, NGC 3147, NGC 3162, NGC 3169, NGC 3198, NGC 3338, NGC 3359. The size is $\sim 3 \times 3$ arcmin. North is up, east to the left. This figure is only available in electronic form at http://www.edpsciences.org

$\chi^{2}$-minimization can be computed from the curvature matrix in the Levenberg-Marquardt algorithm and are very small. The relevant errors here are systematic errors: e.g. non-correct sky-subtraction, non-uniformness of the sky, errors in the determination of the seeing point spread
Fig. 5. $J$-filter images and the residua of the galaxies \#21 to \#30 of our sample: NGC 3368, NGC 3430, NGC 3626, NGC 3675, NGC 3705, NGC 3726, NGC 3810, NGC 3898, NGC 4051, NGC 4254. The size is $\sim 3 \times 3$ arcmin. North is up, east to the left. This figure is only available in electronic form at http://www.edpsciences.org

function, irregular perturbations of the light distribution in the galaxy, etc.

To estimate the error contributions of all these effects, artifical galaxy images with typical sky levels, shot noise and seeing convolution were tested by fitting our 2-dim 
Table 4. Structural parameters of the bulges. $S B_{\text {eff }}$ is the surface brightness in mag/square-arcsec at the effective (half-light) radius $R_{\mathrm{b}} . R_{\mathrm{b}}$ is given in arcsec and in kpc. $\mathrm{PA}_{b}$ is the position angle of the (projected) major axis of the bulge, $Q_{\mathrm{b}}=b / a$ is the axis ratio, and $\beta$ is the slope exponent of the model fit to the bulge. Each value is given for the corresponding filters $J H K$

\begin{tabular}{|c|c|c|c|c|c|c|c|c|c|c|c|c|c|c|c|c|c|c|c|}
\hline No. & NGC & $\begin{array}{c}. S B_{\text {eff }} . \\
J \\
\end{array}$ & $\begin{array}{c}\text { (mag } \\
H\end{array}$ & $\begin{array}{l}\text { sas) } \\
K \\
\end{array}$ & $\begin{array}{l}R_{\mathrm{b}} \\
J\end{array}$ & $\begin{array}{l}\text { csec) } \\
H\end{array}$ & $K$ & $\begin{array}{l}R_{\mathrm{b}} \\
J\end{array}$ & $\begin{array}{c}\mathrm{kpc}) \\
H \\
\end{array}$ & $K$ & $\begin{array}{c}\mathrm{PA}_{\mathrm{b}} \\
J \\
\end{array}$ & (deg) & $K$ & $J$ & $\begin{array}{c}Q_{\mathrm{b}} \\
H \\
\end{array}$ & $K$ & $J$ & $\begin{array}{l}\beta \\
H \\
\end{array}$ & $K$ \\
\hline 1 & 278 & 17.24 & 16.68 & .83 & 3. & 6 & 04 & 0 & 0.44 & 6 & 80 & 10 & 25 & 33 & 0.82 & 33 & 76 & 0.71 & 0.74 \\
\hline 2 & 628 & & 18.06 & & $1:$ & & & & 49 & & & & & 5 & 0.95 & & 71 & 70 & \\
\hline 3 & 772 & 20.50 & & 19.48 & 41.72 & & 34.15 & 6.77 & & 5.54 & 123.90 & & 123.35 & 0.77 & & 79 & 19 & & 18 \\
\hline 4 & 1023 & 3.20 & 16.47 & 17.26 & 3.45 & 18.88 & 31.04 & 1.80 & 1.02 & & 88.46 & 83.98 & 86.89 & 0.78 & 0.78 & 0.78 & 0.22 & 0.29 & 0.21 \\
\hline 5 & 2196 & 18.62 & & 17.94 & 14.98 & & 16.25 & 3.16 & & 3.43 & 56.16 & & 59.13 & 0.78 & & 0.79 & 0.31 & & 0.32 \\
\hline 6 & 2655 & 20.12 & 19.67 & 18.93 & 65.99 & 77.35 & 55.88 & 9.76 & 11.44 & & 85.88 & 85.65 & 89.05 & 0.77 & 0.76 & 0.74 & 0.17 & 0.15 & 0.17 \\
\hline 7 & 2742 & 18.36 & & 17.18 & 2.62 & & 2.68 & 0.38 & & 8 & 87.38 & & & 0.75 & & 76 & 1.40 & & 38 \\
\hline 8 & 2775 & 9.13 & 18.43 & 18.09 & 32.27 & 31.16 & 26.32 & 2 & 3.21 & 71 & 156.00 & 3.88 & 162 & 0.89 & 0.88 & 86 & 25 & 0.23 & 0.25 \\
\hline 9 & 2782 & 8.13 & 16.83 & 16.68 & 7.17 & 5.43 & & & 0.87 & & 1.11 & 111.23 & 108.81 & 0.73 & 0.80 & 0.77 & 0.29 & 0.33 & 0.32 \\
\hline 10 & 2811 & 16.59 & & 15.62 & 5.97 & & 6.20 & 1.22 & & 1.27 & 20.65 & & 20.97 & 0.71 & & 0.69 & 0.41 & & .39 \\
\hline 11 & 2841 & .74 & 17.19 & 17.09 & 4.63 & 26.35 & 26. & 2.97 & 3.18 & & 3.02 & 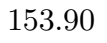 & & 70 & 0.67 & & 0.28 & 0.26 & .26 \\
\hline 12 & 2855 & 18.63 & & 18.23 & 16.67 & & 23.48 & 2.66 & & 4 & 121.34 & & & 35 & & 7 & 0.37 & & .29 \\
\hline 13 & 2964 & 6.31 & & 14.84 & 1.78 & & 6 & 0.24 & & & 88.18 & & & 0.89 & & 34 & 0.58 & & 50 \\
\hline 14 & 2985 & 17.73 & 17.03 & 17.23 & 12.08 & 12.81 & 14.06 & 1.64 & 1.74 & 1.91 & 171.46 & & 178.00 & 0.89 & 0.83 & 6 & 0.35 & 0.35 & 0.33 \\
\hline 15 & 3147 & 6.78 & & 16.25 & 5.87 & & 6.81 & 1.39 & & & 88.79 & & & 0.85 & & 7 & 0.40 & & 0.28 \\
\hline 16 & 162 & .57 & & 17.41 & 3.44 & & 3.26 & 0.47 & & & 8.13 & & & & & & 0.34 & & 36 \\
\hline 17 & 169 & .40 & & 15.60 & 8.53 & & 8.92 & 0.93 & & & 50.08 & & & 0 & & & 0.49 & & 39 \\
\hline 18 & 198 & 19.92 & & 18.64 & 14.70 & & 12.29 & 1.03 & & & 41.23 & & 00 & 0.86 & & 1 & 0.35 & & .31 \\
\hline 19 & 338 & 19.84 & 18.81 & 18.52 & 9. & $0.2 x$ & 7 & 1.26 & 1.20 & & 99.12 & 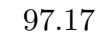 & & 0.80 & 0.80 & 6 & 0.23 & 0.28 & 28 \\
\hline 20 & 59 & 9.62 & & 18.43 & 25.90 & & 23.21 & 2.84 & & & 35 & & & 0.28 & & & 0.72 & & 89 \\
\hline 21 & 368 & .08 & 15.12 & 15.18 & 9.19 & 7.61 & 8.81 & 0.46 & 0.38 & & 9.15 & 150.71 & & 0.75 & 0.78 & 0.79 & 0.60 & 0.68 & 0.56 \\
\hline 22 & 430 & 18.15 & & 16.68 & 2.65 & & 2.18 & 0.43 & & & 24.71 & & & 0.63 & & & 0.80 & & 1.41 \\
\hline 2 & 3626 & 5.19 & & 14.06 & 3.06 & & 2.79 & 0.52 & & & 6.86 & & 57.80 & 0.61 & & & 0.60 & & .62 \\
\hline 24 & 675 & .30 & 16.46 & 16.42 & 0.00 & 9.79 & 10.51 & 0.98 & 0.96 & & 7.24 & 179.0 & 4.18 & 0.61 & 0.65 & 4 & 0.41 & 0.43 & 0.41 \\
\hline 25 & 705 & 19.05 & & 18.02 & 16.40 & & 15.10 & 1.75 & & 1 & 148.21 & & 132.21 & 0.95 & & & 0.27 & & .24 \\
\hline 26 & 726 & 3.01 & & 17.08 & 525 & & 5.40 & 0.51 & & & 24.69 & & & 69 & & & 0.84 & & .00 \\
\hline 27 & 810 & 17.76 & 16.24 & 15.92 & 3.48 & 0.10 & 2.72 & 0.36 & 0.32 & & 14.71 & 7.40 & 11.54 & 0.80 & 0.81 & 0.81 & 0.70 & 1.52 & 1.43 \\
\hline 2 & 898 & 7.86 & & 17.21 & 16.57 & & 17.65 & 3.01 & & & 107.23 & & & 0 . & & 7 & 0.26 & & .23 \\
\hline 2 & 51 & 70 & & $1:$ & 2. & & 4 & 0.26 & & & 138.15 & & & 0.65 & & & 0.36 & & .32 \\
\hline 30 & 254 & 19.90 & 18.99 & 19.25 & 30.86 & 26.75 & 34.24 & 3.10 & 2.68 & & 68.05 & 62.88 & & 0.80 & 0.80 & 0.80 & 0.37 & 0.38 & 0.32 \\
\hline 31 & 303 & 15.58 & & 14.48 & 3.23 & & 3.19 & 0.32 & & & 18.46 & & & 0.97 & & & 0.83 & & 0.94 \\
\hline 3 & 4 & .75 & & & 3. & & & 0.32 & & & 155.77 & & 61 & & & & & & .44 \\
\hline 38 & 0 & 17.55 & & 16.91 & 8.93 & & 9.88 & 0.90 & & & 176.32 & & 0.61 & 0.84 & & & 0.32 & & 0.28 \\
\hline 34 & 501 & 17.78 & 16.46 & 16.32 & 12.50 & 8.67 & 8.89 & 1.25 & 0.87 & 0.89 & 142.14 & .84 & 145.78 & 0.69 & 0.70 & 0.70 & 0.30 & 0.34 & 0.31 \\
\hline 35 & 4535 & 18.26 & & 16.08 & 5.78 & & 3.35 & 0.47 & & & 25.19 & & 84 & 0.83 & & 88 & 0.24 & & 0.33 \\
\hline 3 & 725 & .92 & 16.18 & 3 & & & & 0. & 0.4 & & 1.55 & 169.18 & & 0.91 & 0.89 & 0.90 & 0.46 & 0.48 & 0.47 \\
\hline 37 & 826 & 15.44 & 14.67 & 14.35 & 5.96 & 6.50 & 6.06 & 0.22 & 0.24 & 0.22 & 109.50 & 110.68 & 11 & 0.73 & 0.72 & 0.70 & 0.56 & 0.52 & 0.57 \\
\hline 38 & 5248 & 18.26 & & 17.02 & 18.52 & & 14.74 & 2.04 & & & 110.20 & & & 0.75 & & & 0.43 & & 0.51 \\
\hline 39 & 364 & 19.08 & & 18.22 & 6.01 & & 6.20 & 0.73 & & & 4.15 & & & & & 86 & 0.65 & & 0.67 \\
\hline 40 & 371 & 6.82 & & 15.80 & 4.51 & & 4.27 & 0.99 & & & 46.88 & & & 0. & & 37 & 0.62 & & 0.60 \\
\hline 41 & 371 & 5.78 & & 16.07 & 4.13 & & 4.55 & 0.91 & & 1. & 42.00 & & 40.32 & 0.96 & & 0.84 & 0.62 & & 0.59 \\
\hline
\end{tabular}

Fig. 6. $J$-filter images and the residua of the galaxies \#31 to \#40 of our sample: NGC 4303, NGC 4414, NGC 4450, NGC 4501, NGC 4535, NGC 4725, NGC 4826, NGC 5248, NGC 5364, NGC 5371. The size is $\sim 3 \times 3$ arcmin. North is up, east to the left. This figure is only available in electronic form at http://www.edpsciences.org

models with different wrong offsets of the sky level, offsets of the PSF, etc. From the comparison of these results the errors of the structural parameters $I_{\mathrm{d}}, R_{\mathrm{d}}, I_{\mathrm{b}}, R_{\mathrm{b}}, \beta$, etc. are estimated to be $<15 \%$.

\section{Results of the fits to the galaxy images}

Table 3 lists all galaxies with the structural parameters for the disks resulting from all our fits. All parameters are given for $J$ and $K$, and for $H$ if available. The central surface-brightness values $S B_{\text {c }}$ (in mag/square-arcsec) were obtained from the (unconvolved) central flux densities $I_{\mathrm{d}}$ of the disks using the photometric calibration. Note that these are the values if the galaxies are seen face on, the inclination is considered in the adopted elliptical geometry and can be calculated from $\cos (i)=Q_{\mathrm{d}}=b / a$. The scale lengths 
Table 5. NIR photometric data from the disk-bulge fits. $M_{\text {tot }}$ is the total absolute luminosity, $M_{\text {disk }}$ is the absolute luminosity of the disk, $M_{\text {bulge }}$ that of the bulge. The last column gives the bulge/disk ratios. Each value is given for the corresponding filters $J H K$ (Table 5 is also available at the CDS via anonymous ftp to cdsarc.u-strasbg.fr (130.79.128.5) or via http://cdsweb.u-strasbg.fr/cgi-bin/qcat?J/A+A/368/16)

\begin{tabular}{|c|c|c|c|c|c|c|c|c|c|c|c|c|c|}
\hline No. & NGC & $J$ & $\begin{array}{c}M_{\text {tot }} \\
H\end{array}$ & $\begin{array}{c}(\mathrm{mag}) \\
K\end{array}$ & $J$ & $\begin{array}{c}M_{\text {disk }} \\
H\end{array}$ & $\begin{array}{c}(\mathrm{mag}) \\
K\end{array}$ & $J$ & $\begin{array}{c}M_{\text {bulge }} \\
H\end{array}$ & $\begin{array}{c}\text { (mag) } \\
K\end{array}$ & $\begin{array}{r}\text { bulge } \\
J\end{array}$ & $\begin{array}{r}\text { disk } \\
H\end{array}$ & $\begin{array}{r}\text { ratio } \\
K\end{array}$ \\
\hline 1 & 278 & -23.27 & -23.99 & -23.94 & -23.22 & -23.93 & -23.88 & -19.90 & -20.77 & -20.71 & 0.047 & 0.054 & 0.054 \\
\hline 2 & 628 & -21.69 & -21.97 & -22.35 & -21.58 & -21.81 & -22.14 & -19.11 & -19.78 & -20.46 & 0.102 & 0.154 & 0.213 \\
\hline 3 & 772 & -24.34 & & -25.18 & -23.69 & & -24.67 & -23.48 & & -24.12 & 0.820 & & 0.606 \\
\hline 4 & 1023 & -23.85 & -24.44 & -24.39 & -23.30 & -24.02 & -23.63 & -22.85 & -23.19 & -23.64 & 0.661 & 0.467 & 1.012 \\
\hline 5 & 2196 & -24.06 & & -24.80 & -23.34 & & -23.91 & -23.29 & & -24.17 & 0.953 & & 1.269 \\
\hline 6 & 2655 & -24.79 & -25.61 & -25.56 & -21.80 & -22.56 & -22.40 & -24.72 & -25.54 & -25.50 & 14.702 & 15.644 & 17.397 \\
\hline 7 & 2742 & -22.86 & & -23.85 & -22.84 & & -23.83 & -18.16 & & -19.41 & 0.013 & & 0.017 \\
\hline 8 & 2775 & -24.65 & -25.06 & -25.00 & -24.28 & -24.57 & -24.52 & -23.31 & -23.95 & -23.89 & 0.408 & 0.569 & 0.558 \\
\hline 9 & 2782 & -22.81 & -23.57 & -23.85 & -22.32 & -23.09 & -23.35 & -21.71 & -22.44 & -22.76 & 0.571 & 0.550 & 0.583 \\
\hline 10 & 2811 & -24.11 & & -25.06 & -23.61 & & -24.50 & -23.02 & & -24.06 & 0.579 & & 0.670 \\
\hline 11 & 2841 & -25.51 & -26.12 & -26.35 & -25.16 & -25.73 & -26.01 & -24.13 & -24.82 & -24.92 & 0.389 & 0.432 & 0.367 \\
\hline 12 & 2855 & -23.56 & & -24.52 & -22.69 & & -22.93 & -22.92 & & -24.23 & 1.234 & & 3.308 \\
\hline 13 & 2964 & -22.89 & & -23.91 & -22.82 & & -23.82 & -19.82 & & -21.18 & 0.063 & & 0.088 \\
\hline 14 & 2985 & -23.98 & -24.57 & -24.72 & -23.41 & -23.87 & -24.08 & -23.01 & -23.77 & -23.83 & 0.687 & 0.906 & 0.793 \\
\hline 15 & 3147 & -25.10 & & -25.88 & -24.82 & & -25.51 & -23.49 & & -24.53 & 0.293 & & 0.405 \\
\hline 16 & 3162 & -22.03 & & -22.94 & -21.95 & & -22.84 & -19.26 & & -20.27 & 0.084 & & 0.094 \\
\hline 17 & 3169 & -23.60 & & -24.48 & -23.00 & & -23.70 & -22.68 & & -23.75 & 0.742 & & .050 \\
\hline 18 & 3198 & -22.48 & & -23.18 & -22.40 & & -23.08 & -19.59 & & -20.50 & 0.075 & & 0.093 \\
\hline 19 & 3338 & -22.73 & -23.66 & -23.22 & -22.59 & -23.53 & -23.03 & -20.43 & -21.24 & -21.20 & 0.137 & 0.122 & 0.185 \\
\hline 20 & 3359 & -22.99 & & -24.22 & -22.87 & & -24.13 & -20.50 & & -21.44 & 0.113 & & 0.084 \\
\hline 21 & 3368 & -23.01 & -23.59 & -23.78 & -22.71 & -23.31 & -23.44 & -21.46 & -21.99 & -22.36 & 0.317 & 0.296 & 0.369 \\
\hline 22 & 3430 & -22.50 & & -23.24 & -22.47 & & -23.20 & -18.72 & & -19.60 & 0.032 & & 0.036 \\
\hline 23 & 3626 & -23.77 & & -24.58 & -23.47 & & -24.25 & -22.20 & & -23.13 & 0.309 & & 0.354 \\
\hline 24 & 3675 & -23.75 & -24.50 & -24.64 & -23.55 & -24.28 & -24.40 & -21.83 & -22.67 & -22.88 & 0.207 & 0.227 & 0.247 \\
\hline 25 & 3705 & -23.02 & & -23.87 & -22.57 & & -23.41 & -21.86 & & -22.72 & 0.522 & & 0.528 \\
\hline 26 & 3726 & -23.03 & & -23.73 & -23.00 & & -23.70 & -19.14 & & -20.02 & 0.029 & & 0.034 \\
\hline 27 & 3810 & -22.31 & -23.42 & -23.42 & -22.24 & -23.37 & -23.36 & -19.22 & -20.14 & -20.18 & 0.062 & 0.051 & 0.053 \\
\hline 28 & 3898 & -24.64 & & -25.17 & -23.72 & & -23.55 & -24.03 & & -24.89 & 1.331 & & 3.432 \\
\hline 29 & 4051 & -23.08 & & -23.65 & -22.95 & & -23.50 & -20.67 & & -21.41 & 0.122 & & 0.146 \\
\hline 30 & 4254 & -23.21 & -23.84 & -23.96 & -22.74 & -23.39 & -23.37 & -22.06 & -22.67 & -23.02 & 0.535 & 0.516 & 0.723 \\
\hline 31 & 4303 & -23.72 & & -24.51 & -23.61 & & -24.38 & -21.14 & & -22.13 & 0.102 & & 0.126 \\
\hline 32 & 4414 & -23.35 & & -24.36 & -23.21 & & -24.25 & -21.05 & & -21.85 & 0.136 & & 0.110 \\
\hline 33 & 4450 & -23.52 & & -24.52 & -23.26 & & -24.27 & -21.86 & & -22.79 & 0.276 & & 0.256 \\
\hline 34 & 4501 & -24.43 & -25.14 & -25.38 & -24.29 & -25.02 & -25.26 & -22.16 & -22.65 & -22.89 & 0.141 & 0.112 & 0.112 \\
\hline 35 & 4535 & -23.02 & & -23.83 & -22.97 & & -23.78 & -19.69 & & -20.60 & 0.049 & & 0.054 \\
\hline 36 & 4725 & -22.70 & -23.37 & -23.60 & -22.44 & -23.09 & -23.35 & -21.05 & -21.74 & -21.87 & 0.278 & 0.288 & 0.257 \\
\hline 37 & 4826 & -22.87 & -23.62 & -23.69 & -22.74 & -23.46 & -23.53 & -20.48 & -21.47 & -21.56 & 0.125 & 0.160 & 0.164 \\
\hline 38 & 5248 & -24.47 & & -25.40 & -24.25 & & -25.23 & -22.66 & & -23.33 & 0.232 & & 0.174 \\
\hline 39 & 5364 & -23.22 & & -24.39 & -23.19 & & -24.36 & -19.55 & & -20.47 & 0.035 & & 0.028 \\
\hline 40 & 5371 & -24.94 & & -25.77 & -24.83 & & -25.66 & -22.33 & & -23.28 & 0.099 & & 0.112 \\
\hline 41 & 5371 & -24.89 & & -25.89 & -24.77 & & -25.79 & -22.46 & & -23.27 & 0.120 & & 0.098 \\
\hline
\end{tabular}

$R_{\mathrm{d}}$ are given in arcsec and in $\mathrm{kpc}$, using the distances from Table 1. The last columns give the position angles $\mathrm{PA}_{\mathrm{d}}=\Phi_{\mathrm{d}}$, and the axis ratios $Q_{\mathrm{d}}=b / a$.

Table 4 lists the corresponding structural parameters for the bulges, for $J$ and $K$, and for $H$ if available. The $S B_{\text {eff }}$ numbers were calculated from the $I_{\mathrm{b}}$ values via the photometric calibration. The effective radii $R_{\mathrm{b}}$ are given again in arcsec and in $\mathrm{kpc}$, using the distances from Table 1 . The last columns give the position angles $\mathrm{PA}_{\mathrm{b}}=\Phi_{\mathrm{b}}$, the axis ratios $Q_{\mathrm{b}}=b / a$, and the bulge exponent $\beta=1 / n$.
From the parameter values of $I_{\mathrm{d}}, R_{\mathrm{d}}$, it is very easy to calculate the integrated flux for the disks by integration from the centre to infinity. No external truncation radius was used here. In a similar manner the bulge flux was integrated with the values of $I_{\mathrm{b}}, R_{\mathrm{b}}, \beta$ (see Caon et al. 1993). The total flux is simply the sum of the fluxes of disk and bulge. The bulge-disk ratio is the corresponding quotient. The absolute magnitudes were calculated using the distances from Table 1. Table 5 lists all the photometrical parameters from the disk-bulge fits in $J H K$. 


\subsection{Comparison of the results in JHK}

The structural parameters generally have similar values in the different filters $J H K$. We consider the ratios $R_{\mathrm{d}}(J) / R_{\mathrm{d}}(K), R_{\mathrm{b}}(J) / R_{\mathrm{b}}(K)$, and $\beta(J) / \beta(K)$. There is no significant trend of the corresponding ratios with the values of $R_{\mathrm{d}}$ or $\beta$. Only $R_{\mathrm{b}}(J) / R_{\mathrm{b}}(K)$ shows an increase to low $R_{\mathrm{b}}$ values $\left(R_{\mathrm{b}}<7^{\prime \prime}\right)$ which is probably a consequence of the larger errors due to the limited resolution there.

The ratios of the structural parameters are only very weakly dependent on the Hubble type. There is a weak tendency of the ratios of $R_{\mathrm{b}}$ to increase, and of the ratios of $\beta$ to decrease with the Hubble types. However, the gradients of the corresponding regressions are very small. The mean value of the quotients and their rms variations are $R_{\mathrm{d}}(J) / R_{\mathrm{d}}(K)=1.01 \pm 0.19, R_{\mathrm{b}}(J) / R_{\mathrm{b}}(K)=1.09 \pm 0.26$, $\beta(J) / \beta(K)=1.01 \pm 0.16$. This means that $R_{\mathrm{d}}, R_{\mathrm{b}}, \beta$ are practically identical for $J$ and $K$, the scatter is consistent with our error estimate in Sect. 4.5.

The value of the color index $J-K$ shows a weak increase with Hubble type We neglect this hardly significant gradient and calculate the mean color indices from our model flux integrations to $J-K=0.84 \pm 0.18$ and correspondingly $J-H=0.71 \pm 0.19$.

\subsection{Remarks to individual objects}

For some of our sample galaxies there exists a large number of papers. The purpose of this section is not to give a review over the most important results of all this research, but to present those aspects which are relevant for our fit and disk/bulge decomposition. All Hubble types used in this paper were taken from Sandage \& Bedke (1994).

1. NGC 278 is a face-on multiple-armed Sbc galaxy $(T=4)$. On HST images Phillips et al. (1996) could follow the spiral structure to $2-3$ arcsec from the centre. The fits of the surface-brightness $(S B)$ distributions in $J H K$ lead to similar results for the structural parameters (Tables 3 and 4). The residuum shows remnants of the spiral arms and of dust filaments, but looks good concerning the fit quality (Fig. 3). The exact distance determination for NGC 278 is a problem, since the small radial velocity is not meaningful for that. We use the mean value of the discordant distance values from Tully (14.74 Mpc) and Bottinelli et al. (32.65 Mpc).

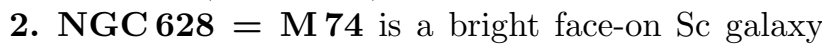
$(T=5)$. The distance of NGC 628 was individually determined by Sharina et al. (1996) to $D=7.80 \mathrm{Mpc}$. Our fit results for $R_{\mathrm{d}}, R_{\mathrm{b}}, \beta$ in $J H K$ are similar to each other, however with quite a scatter (Tables 3,4 ). The reason is probably the large diameter of the galaxy compared to our field of view $(3 \times 3$ arcmin $)$. Nevertheless, the residua of our fit look excellent (Fig. 3). A comparison with the fit results of other authors will be made in the next section.

3. $\mathbf{N G C ~} 772=\operatorname{Arp} 78$ is an Sb galaxy with one especially strong spiral arm. Nevertheless the fit of the disk works fairly good and shows very low residua.
4. NGC 1023 is one of the few S0 (SB0) galaxies in our sample. It has a very good distance determination (11.11 Mpc) by Ferrarese et al. (2000). The residua show a dipole structure near the centre, probably due to the central nuclear disk (Sil'chenko 1999) which we did not consider in the fit procedure. From our disk-bulge decomposition we obtained a half-light radius of the bulge of 30 to $35^{\prime \prime}$ which is in fairly good agreement with the corresponding bulge radius obtained from stellar velocity dispersion profiles ( $\approx 50^{\prime \prime}$, Simien \& Prugniel 1997).

5. NGC 2196 is a nearly face-on $\mathrm{Sa} / \mathrm{Sab}$ galaxy. Carollo \& Stiavelli (1998) found on HST images the continuation of the $R^{1 / 4}$ density profile of the bulge to less than $1^{\prime \prime}$ radius. We obtained a slope of $\beta=0.31$ for the whole bulge. The residua of our disk-bulge fit look good. The star near the centre was extracted before the fit.

6. $\mathbf{N G C ~} 2655=\operatorname{Arp} 225$ is an Sa galaxy which shows traces of a strong interaction or merger event: faint outer stellar loops, extended HI-envelope (Huchtmeier \& Richter 1982). The bulge in NGC 2655 is especially large (Table 4). The central dust-structure (Erwin et al. 1996) is probably the reason for the disturbed fit residuum there.

7. NGC 2742 is a very regular Sc galaxy of medium inclination. The residuum image (Fig. 3) shows the arms as positive features (white) and the inter-arm regions as negative residuals (black).

8. NGC 2775 is an Sa galaxy of low inclination. The nearly perfect residuals show nicely the fine structure of the highly multiple arms (Fig. 3). Corsini et al. (1999) measured the profile of the stellar velocity dispersion of the bulge of NGC 2775: their radius of $\approx 50^{\prime \prime}$ coincides well with our value $\left(32^{\prime \prime}\right)$ for the effective radius.

9. $\mathbf{N G C 2 7 8 2}=\operatorname{Arp} 215$ is an isolated face-on $\mathrm{Sa}$ galaxy showing HI gas plumes extending $5^{\prime}$ to the east and $2^{\prime}$ to the northwest (Smith 1991), probably formed during a merger event. The eastern plume is associated with a stellar tail of similar size. However, this feature is outside our $3^{\prime}$ field of view. The main disk of NGC 2782 is fairly undisturbed (Jogee et al. 1998). Our residuum shows some irregularities near the centre, which are probably due to the strong central star-formation region (Boer et al. 1992; Jogee et al. 1998, 1998; Yoshida et al. 1999).

10. NGC 2811 is an Sa galaxy of medium inclination. The bulge has a slightly boxy shape which leads to the dipole structure in the residuum.

11. NGC 2841 is an Sb galaxy of medium inclination and with regular, flocculent arms. Using the HST Cepheid distances and SN Ia distances Shanks (1997) determined the distance of NGC 2841 to $24.9 \mathrm{Mpc}$. In the central regions there exist a slight rotation of the isophotes' major axis, this is responsible for the double-cone feature in the residuum image (Fig. 4 in on-line version). The reason is probably a weak bar component (Keel 1983; Varela et al. 1996; Afanasiev \& Sil'chenko 1999).

12. NGC 2855 is an Sa galaxy of low inclination. It is surrounded by faint stellar shells which may be a remnant of an interaction. The filamentary dust arms visible in 
optical images disappear in the NIR. The residuum looks very good.

13. NGC 2964 is an Sc galaxy with strong massive arms. It may form a physical pair with the S0 galaxy NGC 2968 at a separation of $6.2^{\prime}$ (Sandage \& Bedke 1994). However, the spiral pattern is fairly regular.

14. NGC 2985 is an Sab spiral with very regular, tight arms. The fit procedure worked very good, the corresponding low residuum is shown in Fig. 4 (in on-line version).

15. NGC 3147 is an $\mathrm{Sb}$ spiral of low inclination $\left(Q_{\text {disk }}=0.84, \mathrm{PA}_{\text {disk }}=150^{\circ}\right)$. The bulge has a similar axis ratio, but appears at a very different position angle $\mathrm{PA}_{\text {bulge }}=88^{\circ}$. Since it is not plausible that the different orientations of the (projected) major axes of disk and bulge are due to a different plane of symmetry, the bulge must be triaxial. NGC 3147 is one of the rare examples with such a feature. Most triaxial bulges seen on visual images are artefacts of dust absorption and disappear in the NIR.

16. NGC 3162 is an Sbc spiral of low inclination. One arm is prominent, the other one much weaker. This could be a consequence of an interaction. Nevertheless we obtained a good fit with good residua (Fig. 4 in on-line version).

17. NGC 3169 is an Sb spiral of medium inclination, it forms a pair with NGC 3166 at $7.7^{\prime}$ angular distance. There exist signs of interaction (warps; Sandage \& Bedke 1994). The strong dust lane is still visible in the $J$ image, especially in the residuum (Fig. 4 in on-line version).

18. NGC 3198 is an Sc spiral of fairly high inclination, showing numerous HII regions (Corradi et al. 1991). In the HST key project the Cepheid distance of NGC 3198 was determined to $14.5 \mathrm{Mpc}$ (Kelson et al. 1999).

19. NGC 3338 is an Sbc spiral of medium inclination, it has thin and well ordered arms. Correspondingly the fit procedure yielded a very low residuum (Fig. 4 in on-line version).

20. NGC 3359 is a nearly face-on SBc spiral with a fairly weak bar and practically no bulge. Thus our disk/bulge fit treats the bar as a bulge of high ellipticity (see the $Q_{\mathrm{b}}$ in Table 2) and works still satisfying (Fig. 4). However, the residuum is asymmetric due to the slightly banana-shaped light distribution in the bar.

21. NGC $3368=$ M96 is an Sab spiral of low inclination. It is the brightest galaxy in a group of galaxies in Leo (e.g. Schneider 1989). Our field of view is a bit too small for this object. Due to a complex substructure and isophote twist in the central region, our fit yields only fairly good residua (may be double bar, see Jungwiert et al. 1997). In the HST key project the Cepheid distance of NGC 3368 was determined to $11.2 \mathrm{Mpc}$ (Tanvir et al. 1999; Gibson et al. 2000).

22. NGC 3430 is a fairly regular Sbc spiral with thin arms and with medium inclination. The fits yielded excellent residua (Fig. 5 in on-line version).

23. NGC 3626 is an Sa spiral of medium inclination. Ciri et al. (1995) found that the gas in this galaxy is counter-rotating to the stars. This is probably the remnant of a merging or accretion event. NGC 3626 shows a prominent dust lane (ring) around the central bulge. The fit residuum shows the corresponding substructure there.

24. NGC 3675 is an Sb spiral of fairly high inclination. The circular dust near the centre (very good detectable in a $\mathrm{Pa}_{\alpha}$ snapshot image of the HST, Böker et al. 1999) leads to some problems with the fit there; this is visible in the residuum (Fig. 5 in on-line version). Eskridge et al. (2000) classify NGC 3675 as strongly barred from their analysis of NIR images in the $H$ filter. We found a bulge component with the same position angle as the disk and somewhat rounder in projection $\left(Q_{\mathrm{b}}=0.61\right.$, $\left.Q_{\mathrm{d}}=0.43\right)$, but no signature of a bar.

25. NGC 3705 is a regular Sb spiral of medium inclination and with thin outer arms. A bright star $\approx 9^{\prime \prime} \mathrm{NW}$ of the centre disturbs the residuum. This star was masked for the fitting procedure.

26. NGC 3726 is an Sbc spiral of medium inclination. It has a small weak bar (Martin 1995). Nevertheless the fit was satisfying and lead to good residua (Fig. 5 in on-line version).

27. NGC 3810 is an Sc spiral of low inclination. It is characterized by a fairly sudden decrease of surface brightness between inner and outer arms, without a change of position angle. However, the fit did not pose any problems.

28. NGC 3898 is an Sa spiral of medium inclination with multiple thin arms. Carollo \& Stiavelli (1998) found on HST images the continuation of the $R^{1 / 4}$ density profile of the bulge to less than $1^{\prime \prime}$ radius.

29. NGC 4051 is an Sbc spiral of medium inclination. It is a well investigated Seyfert galaxy. The light contribution of the active nuclues was not considered in the fit, a corresponding tiny residuum can be seen in Fig. 5 (in on-line version).

30. NGC $4254=$ M 99 is a face-on Sc grand design spiral in the Virgo cluster. The western $m=1$ mode arm is not fully covered by our field of view. Since the galaxy does not show any other peculiar features in NIR, the fit procedure lead to good results.

31. NGC $4303=$ M 61 is a face-on Sc spiral in the Virgo cluster. It has a weak bar in NS direction (RC3: Type $\mathrm{SAB}(\mathrm{rs})$ ) which caused no problems for the diskbulge fit. However, a star forming ring of $\approx 6^{\prime \prime}$ diameter (Colina et al. 1997; Colina \& Arribas 1999) produces a clear corresponding signature in the residuum image (Fig. 6 in on-line version).

32. NGC 4414 is a multi-armed flocculent Sc spiral of medium inclination in the Virgo cluster. We obtained good fit residua. In the HST key project the Cepheid distance of NGC 4414 was determined to 19.1 Mpc (Turner et al. 1998).

33. NGC 4450 a Virgo cluster galaxy, is an Sab spiral of medium inclination with soft spiral arms. The galaxy shows an inner structure with a slight different position angle $\left(\Delta \mathrm{PA} \approx 5^{\circ}\right)$ compared to the disk. This may be interpreted as a lens or bar (Rubin et al. 1997; Eskridge et al. 2000). Nevertheless, our fit lead to good results. 
34. NGC $4501=$ M 88 is a prominent Sbc spiral in the Virgo subcluster around NGC 4486. On red HST images Carollo et al. (1998) could follow the spiral structure of NGC 4501 down to the nucleus. Due to the medium high inclination of this galaxy a reasonable fit with our limited field of view was still possible. The arm structure is very regular; we obtained very good residua.

35. NGC 4535 is a large face-on SBc galaxy with two strong arms in the Virgo cluster. Nevertheless, we obtained quite reliable fit results, only the small central bar lead to some problems with the residuum there. In the HST key project the Cepheid distance of NGC 4535 was determined to 16.6 Mpc (Macri et al. 1999; Sakai et al. 2000).

36. NGC 4725 is an $\mathrm{Sb} / \mathrm{SBb}$ spiral of medium inclination. The neglect of the small bar in our fit resulted in a corresponding residual feature (Fig. 6). In the HST key project the Cepheid distance of NGC 4725 was determined to 13.0 Mpc (Gibson et al. 1999; Sakai et al. 2000).

37. NGC $4826=$ M64 is an Sab spiral of medium high inclination. The galaxy shows two counter-rotating gas disks (ionized gas and neutral hydrogen) in the outer region, pointing towards an accretion event (e.g. Braun et al. 1994; Rubin 1994). The strong dust lane (bad eye galaxy, e.g. Walterbos et al. 1994) is still visible even in the NIR, leading to some problems with the fit.

38. NGC 5248 is an Sbc spiral of medium inclination. The outer arms have very low surface brightness and are outside our field of view. NGC 5248 has a central ring of star formation of $\approx 12^{\prime \prime}$ diameter (Storchi-Bergmann et al. 1996). Inside this ring a tiny nuclear spiral was discovered (Laine et al. 1999). These nuclear structures produce a corresponding residuum pattern as result of our disk-bulge fit (Fig. 6 in on-line version).

39. NGC 5364 is a very regular Sc spiral of medium inclination. The regular spirals always give excellent fit results and low residua (Fig. 6 in on-line version).

40. NGC 5371 is an $\mathrm{Sb} / \mathrm{SBb}$ spiral of low inclination. The faint bar (e.g. Martin 1995) is no problem for our fit (Fig. 6 in on-line version).

\subsection{Comparison with previous papers}

Although there exist quite a number of papers about diskbulge decompositions in the literature (see Sect. 1), it is not easy to compare the results. The reasons are that different authors use different detectors/filters, different methods, and different parametrizations of the $S B$ distribution. The choice of a fixed or variable $\beta=1 / n$ is also of great importance for the results.

Only for two galaxies of our sample (NGC 628 and NGC 2841) there exist several studies in the literature measuring the $S B$ distribution in optical or NIR colors.

As an example, we show in Table 6 all available scale lengths for disk and bulge of NGC 628, normalized in arcsec. The blue disk scale lengths of the different authors coincide fairly well, apart from the value of
Table 6. Disk- and bulge parameters of NGC 628 from studies of different authors in different filters

\begin{tabular}{|l|c|r|r|c|}
\hline Author(s) & Color & $\begin{array}{c}R_{\mathrm{d}} \\
\text { arcsec }\end{array}$ & $\begin{array}{c}R_{\mathrm{b}} \\
\operatorname{arcsec}\end{array}$ & $\beta$ \\
\hline Boroson (1981) & phot & 85.72 & 25.53 & 0.25 \\
Grosbøl (1985) & phot & 66.60 & - & - \\
Simien, de V. (1986) & phot & 144.00 & 14.40 & 0.25 \\
Baggett et al. (1998) & phot & 56.2 & 290.48 & 0.25 \\
Lu (1998) & $B$ & 77.60 & - & - \\
\hline Natali et al. (1992) & $U$ & 84.49 & - & - \\
" & $B$ & 68.23 & - & - \\
" & $V$ & 64.86 & - & - \\
" & $R$ & 65.25 & - & - \\
" & $I$ & 65.65 & - & - \\
\hline Moellenhoff (2001) & $U$ & 127.60 & 11.48 & 0.85 \\
" & $B$ & 85.18 & 10.47 & 0.77 \\
" & $V$ & 76.04 & 11.97 & 0.69 \\
" & $R$ & 71.52 & 12.97 & 0.66 \\
" & $I$ & 70.12 & 15.34 & 0.62 \\
\hline This paper & $J$ & 55.70 & 13.41 & 0.71 \\
" & $H$ & 45.38 & 13.04 & 0.70 \\
" & $K$ & 73.08 & 19.50 & 0.60 \\
\hline
\end{tabular}

Simien \& de Vaucouleurs (1986). The differing numbers of Bagget et al. (1998) are due to their different choice of the fit parameters (inner-truncated disk). The red scale length values of Natali et al. (1992) tend to shorter values.

In a recent observational run (Feb. 2000) we used the Calar Alto $2.2 \mathrm{~m}$ telescope with a focal reducer (field of view $16^{\prime}, 0.53^{\prime \prime} / \mathrm{pix}$ ) for an $U B V R I$ imaging of NGC 628 . The structural parameters have been obtained by the methods described in this paper. The large field of view reduces the sky-subtraction problems to a minimum. Our results for $R_{\mathrm{d}}, R_{\mathrm{b}}, \beta$ show the behaviour already described in Möllenhoff et al. (1999): the disk scale length decreases from $U$ to $I$ and $J H K$. However, there is quite a scatter between the NIR values. The large diameter of NGC 628 has the consequence that only minor errors in the sky determination lead to strong variations of the $R_{\mathrm{d}}$-values. The reason for the longer scale length in the blue colors is probably that the stars in the outer regions are younger and have lower metalicity (de Jong 1996c). Nevertheless, the residua of our fit look excellent (Fig. 3). The results of Natali et al. (1992) also show the tendency of a decreasing scale length to redder colors, however less marked. The difference to the results of Möllenhoff (2000) may be due to the fact, that Natali et al. truncated the innermost region and considered only a disk fit.

The bulge of NGC 628 behaves just vice versa (Table 6 ): the half-light radius $R_{\mathrm{b}}$ increases from blue to red (apart from the not very reliable value in $K$ ). The slope decreases as well from blue to red, i.e. in blue colors the bulge is smaller and steeper outsid $R_{\mathrm{b}}$. This is probably a population effect as well (Möllenhoff et al. 1999).

Concerning the comparison of whole samples of different authors for better statistics, the data situation is not very satisfying. Only a minority of the different 

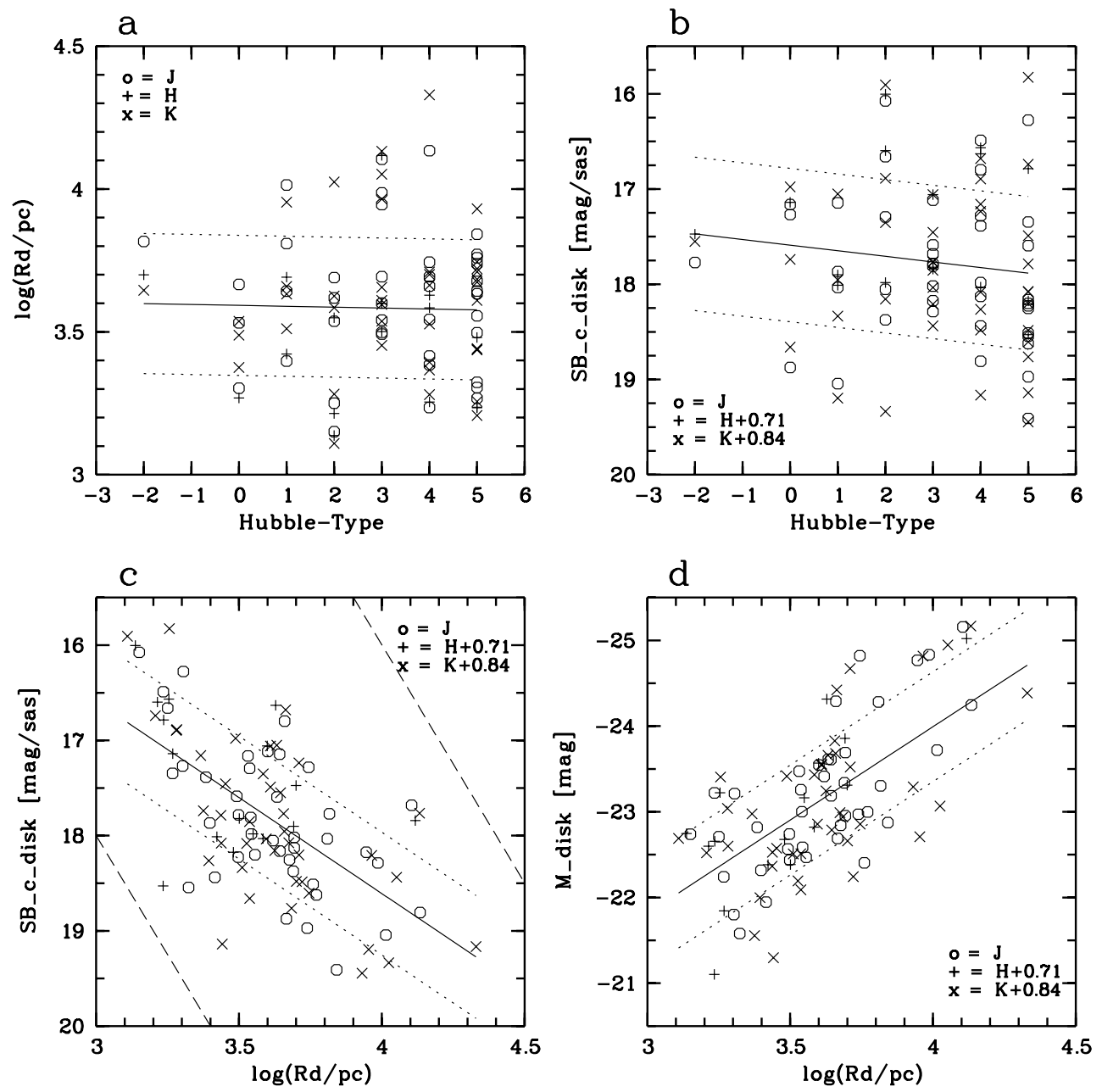

Fig. 7. (a-d) Correlation of disk parameters with Hubble types and with other disk parameters. The different colors are marked with different symbols $(0=J,+=H, \times=K)$. In each plot the solid line represents the linear regression through all corresponding points, the dotted lines represent the $1 \sigma$-deviations. Neither the disk scale-lengths (a) nor the central $S B$ values (b) show any systematic dependency on the Hubble types. However, there exist fairly strong correlations among the disk parameters themselves: Large disks have a lower central surface brightness (c), and large disks have a higher absolute luminosity in NIR (d)

Table 7. Disk- and bulge parameters of NGC 4450 from NIR studies of different authors

\begin{tabular}{|l|c|c|c|c|}
\hline Author(s) & Color & $\begin{array}{c}R_{\mathrm{d}} \\
\operatorname{arcsec}\end{array}$ & $\begin{array}{c}R_{\mathrm{b}} \\
\operatorname{arcsec}\end{array}$ & $\beta$ \\
\hline de Jong (1996) & $B$ & 52.40 & 3.60 & 1.00 \\
" & $K$ & 44.80 & 4.10 & 1.00 \\
\hline Moriondo et al. (1998) & $K$ & 40.30 & 12.42 & 0.33 \\
" & $K$ & 35.37 & 16.31 & 0.25 \\
\hline This paper & $J$ & 34.33 & 8.93 & 0.32 \\
" & $K$ & 38.31 & 9.88 & 0.28 \\
\hline
\end{tabular}

samples have enough galaxies in common. From the old era of photographic plates there exists the big sample of Grosbøl (1985, R POSS plates, disk scale lengths obtained from ellipse fits) which has 29 galaxies in common with ours. The quotient $R_{\mathrm{d}} / R_{\mathrm{d}-\text { Grosbol has a mean value }}$ of 0.90 with a scatter of \pm 0.33 . With Boroson (1981, major- and minor-axis profiles, 1-dim disk-bulge decomposition) we have 7 galaxies in common. The mean

values and scatter are $R_{\mathrm{d}} / R_{\mathrm{d}-\text { Boroson }}=0.85 \pm 0.43$ and $R_{\mathrm{b}} / R_{\mathrm{b}-\text { Boroson }}=1.30 \pm 0.67$. Although the scatter is quite big, all three quotients reflect the findings that the disk scale lengths are larger in blue colors, the bulge effective radii behave vice versa (de Jong 1996b,c; Möllenhoff et al. 1999). Other studies with photographic plates (e.g. Simien \& de Vaucouleurs 1986; Kodaira et al. 1986; Schombert \& Bothun 1987) contain only very few galaxies of our sample.

Baggett et al. (1998) present the results of a 1-dim profile decomposition of a large sample of galaxies, based on digitized photographic plates (Kodaira et al. 1990). They use a combination of a de Vaucouleurs law for the bulge and an inner-truncated exponential disk (Kormendy 1977a). We have 31 galaxies in common with Baggett et al. The results show quite a scatter $\left(R_{\mathrm{d}} / R_{\mathrm{d}-\text { Baggett }}=1.20 \pm\right.$ 0.59 and $R_{\mathrm{b}} / R_{\mathrm{b}-\text { Baggett }}=1.31 \pm 1.87$ ) which is probably a consequence of the different choice of fit parameters.

The more modern CCD studies should generally be of better quality due to the better photometric calibration 

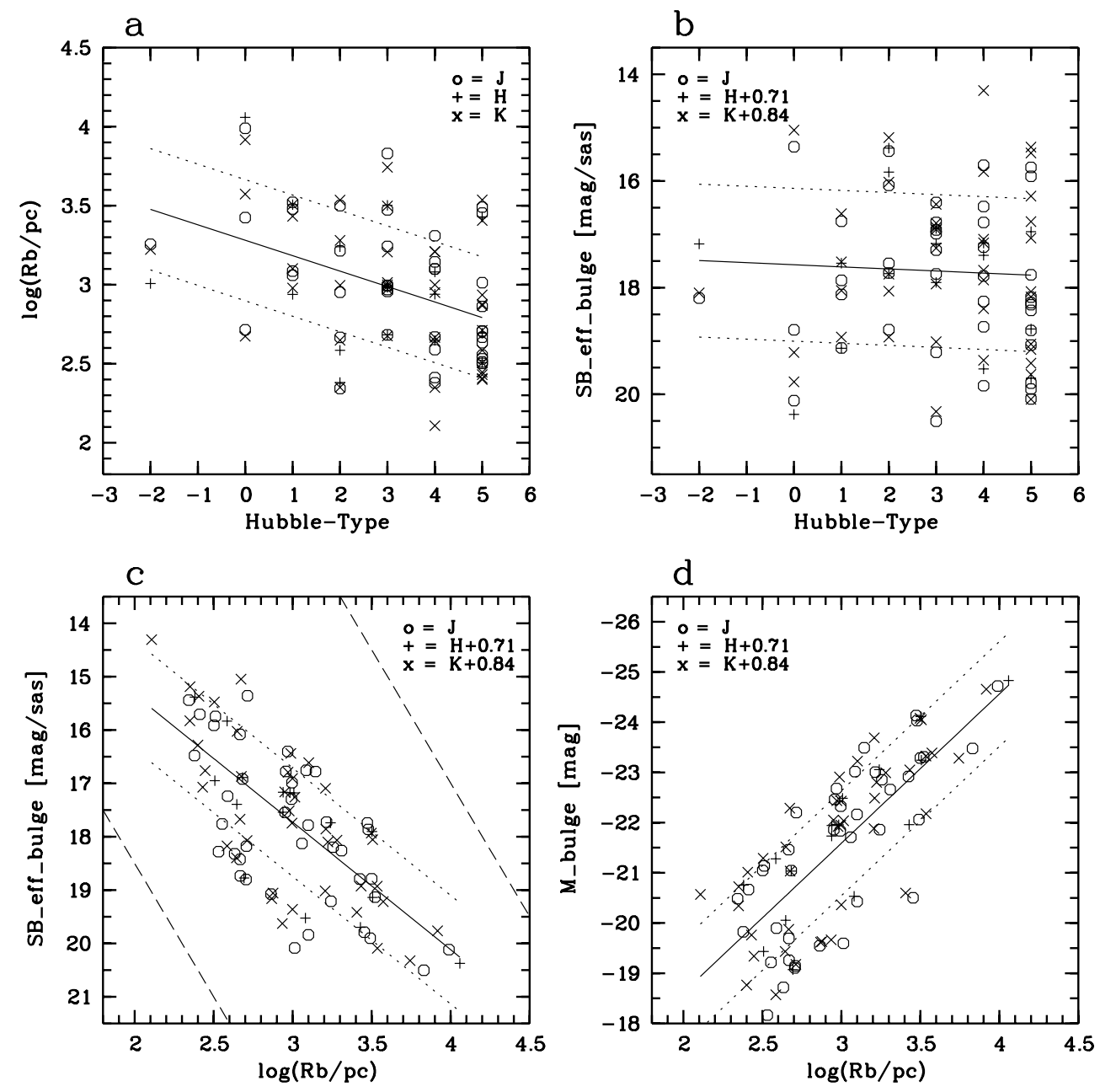

Fig. 8. (a-d) Correlation of bulge parameters, symbols and lines as in Fig. 7. While the bulge effective radii show a weak dependency on Hubble types (a), this is not true for $\mathrm{SB}_{\text {eff }}$, which only shows a big scatter (b). On the other hand, large bulges have a lower $\mathrm{SB}_{\text {eff }}(\mathbf{c})$ and a higher absolute luminosity (d)

of the CCD detectors. However, we oftenly face again the problem of disjunct samples (e.g. Kent 1985; Prieto et al. 1992; Courteau 1996; Pompei \& Natali 1997; Lu 1998). Héraudau \& Simien (1996) presented CCD photometry in $V$ and $I$ of 234 spiral galaxies. We have 17 object in common with them. However, they determined the effective or half-light radii for the whole galaxies, without separation of disks and bulges. For a comparison we calculate the effective radii of our disks ( $J$ filter) according to $R_{\text {eff }}=R_{\mathrm{d}} * 1.6716$ (see Eq. (6)) and compare them with

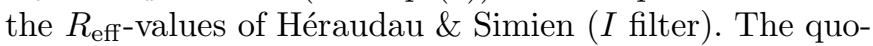
tient $R_{\text {eff }} / R_{\text {eff }-\mathrm{HS}}$ has a mean value of $1.11 \pm 0.55$. Here the influence of strong bulge contributions disturbs the comparison. If we restrict ourselves to those galaxies with bulge/disk-ratios $<0.3$ (10 objects in common) we obtain $0.91 \pm 0.23$ for the mean quotient of the effective radii, i.e. a better agreement.

There exist not yet many NIR studies on disk-bulge decompositions. With de Jong (1996a,b,c) we have only two galaxies in common (NGC 3162 and 4450), with Moriondo et al. (1998) four galaxies (NGC 2775, 2841, 3898, 4450).
Table 7 displays all NIR results for NGC 4450. While the disk scale-lengths $R_{\mathrm{d}}$ are fairly similar, the bulge half-light radii $R_{\mathrm{b}}$ are quite different. They are very sensitive on the choice of the slope $\beta$. If one compares the models with similar $\beta$, the coincidence is more satisfying. This is also valid for the comparison of our model parameters of NGC 2775 and NGC 3898 with those of Moriondo et al. (not shown here).

Andredakis et al. (1995) studied the $S B$ profiles for a $K$-band sample of 30 spirals of fairly high inclination. They used a different fit method, where a 1-dim cut is swept azimuthally by $360^{\circ}$ over the galaxy. They found that a variable $\beta=1 / n$ is advantageous for bulge fits. This was also supported by Seigar \& James (1998), who studied of 45 spirals in $J, K$ with 1-dim disk-bulge fits. Khosroshahi et al. (2000a,b) used the $K$ sample of Andredakis et al. to fit 2-dim $S B$ functions with a variable $\beta=1 / n$. Héraudau et al. (1996) obtained NIR images of 31 southern spirals and made a disk-bulge decomposition of 6 objects, together with kinematical models (Héraudau \& Simien 1997). All these papers have no galaxies in 

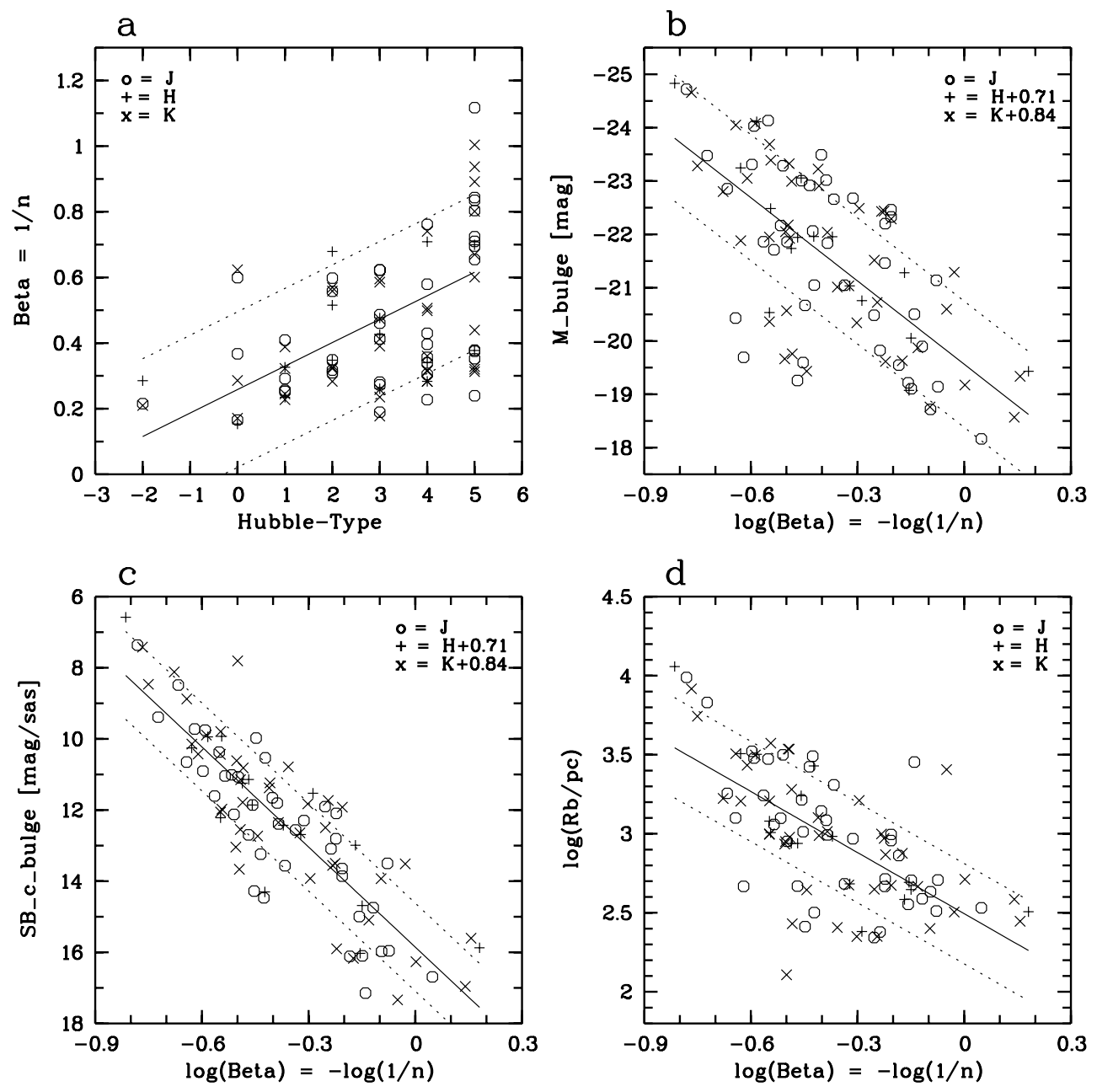

Fig. 9. (a-d) Correlations of the bulge exponent $\beta=1 / n$, symbols and lines as in Fig. 7. The bulge exponent $\beta=1 / n$ increases with the Hubble types, the late types have profiles which decrease faster in the outer radii (a). The NIR absolute luminosity of the bulges decreases with $\beta$ (b). The central $S B$ of the bulges shows a strong anti-correlation with $\beta$ (c). The small bulges have larger $\beta$ values (d)

common with our sample, but it is interesting to compare these results with ours statistically.

\section{Correlations and statistics of structural parameters}

The study of statistical correlations between the structural parameters is an efficient tool, since it offers the possibility to discover systematic relations which give clues to the formation or evolution of spiral galaxies.

The resulting structural parameters of all 40 sample galaxies and their correlations are presented in Figs. 7-11. The different colors are marked with different symbols $(\circ=J,+=H, \times=K)$. While for the geometric parameters like scale length $R_{\mathrm{d}}$ the values of $J H K$ can directly be compared and plotted into the same diagram, this is not possible for the photometric parameters like luminosity $M_{\text {disk }}$ or surface brightness $S B_{\text {c }}$. According to the mean values $J-H=0.71$ and $J-K=0.84$ from Sect. 5.1 we plot $H^{\prime}=H+0.71$ and $K^{\prime}=K+0.84$ together with the $J$ values into the corresponding diagram. In each diagram the continuous line is the linear regression through the corresponding data points of the spiral galaxies, the dotted lines repesent the $1 \sigma$-deviations.

\subsection{Disk parameters}

There are no correlations of the disk parameters with the Hubble types: The disk scale-lengths (Fig. 7a) do not show any systematic dependency (Pearson's correlation coefficient is $r=0.023)$. Moreover, the scale-lengths show a fairly big variation from galaxy to galaxy. This is consistent with results of de Jong (1996b).

A similar result was found for the values of the central surface brightness of the disks, there are only minor signs of a decreasing $S B_{\mathrm{c}}$ with Hubble type (Fig. $7 \mathrm{~b}, r=0.13$ ). This tendency was also quoted by de Jong (1996b). The values of the central $S B$ are not at all constant for the disks of spiral galaxies as was found by Freeman (1970) in photopraphic material, but show a scatter of 3 magnitudes in NIR. 

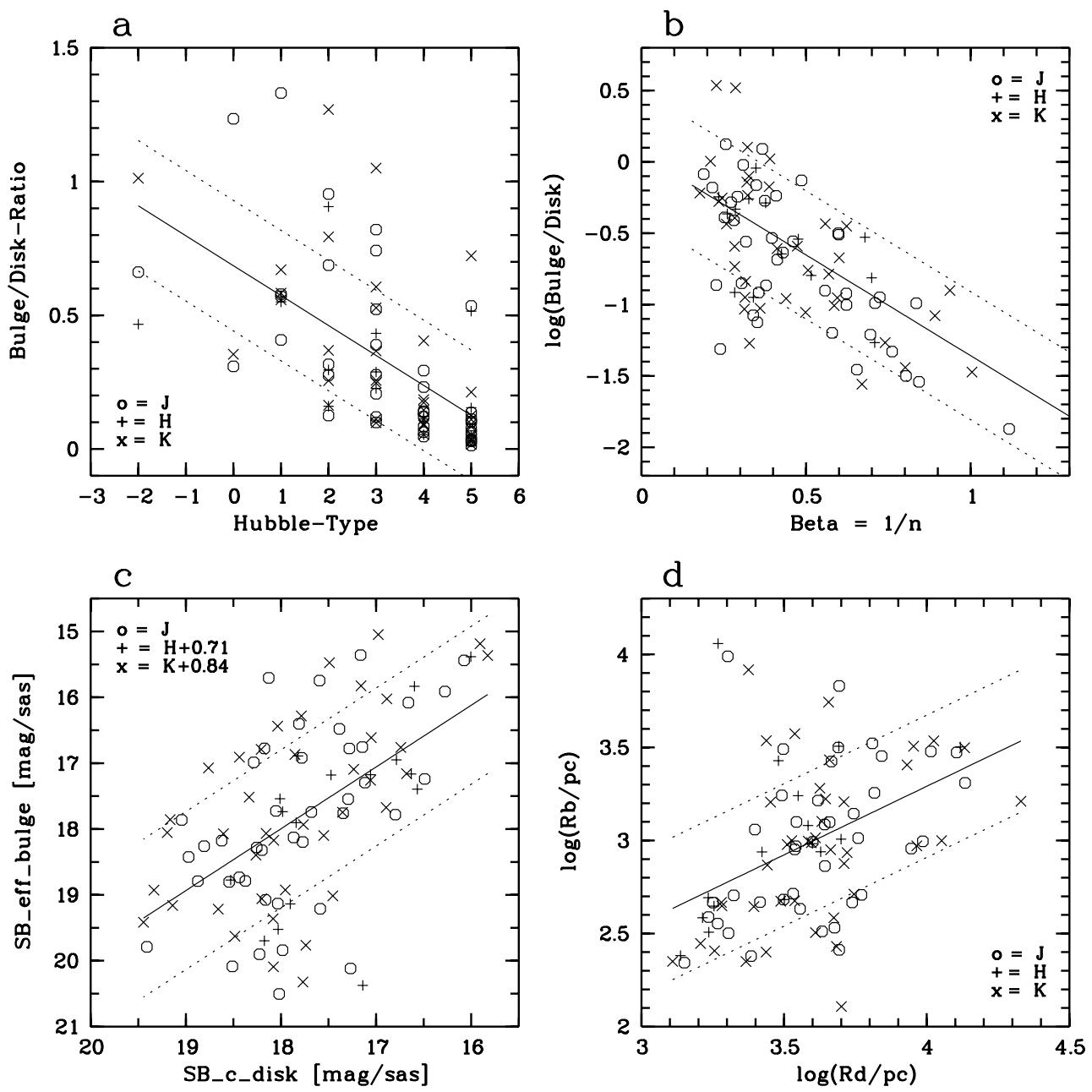

Fig. 10. (a-d) The correlations between disk- and bulge-parameters are only fairly weak (symbols and lines as in Fig. 7). The bulge-disk ratio decreases with Hubble type (a) as well as with $\beta$ (b). The effective $S B$ of the bulges correlates with the central $S B$ of the disks, however with a big scatter (c). There exists a weak correlation between bulge- and disk-radii (d)

However, there exist fairly clear correlations between the disk parameters among themselves: the central surface brightness of the disks shows a statistical significant dependency on the disk scale-length: large disks have a lower central surface brightness. The linear regression is $S B_{\mathrm{c}}=10.54+2.02 \log \left(R_{\mathrm{d}} / \mathrm{pc}\right) \pm 0.64$ with a correlation coefficient $r=0.61$ (Fig. 7c). This relation is not the result of a roughly constant disk luminosity of our sample: the dashed lines in Fig. 7c are lines of constant luminosity, corresponding to the equation $S B_{\mathrm{c}}=$ const. $+5 \log \left(R_{\mathrm{d}} / \mathrm{pc}\right)$. They are much steeper than our relation and demonstrate that the correlation between $S B$ and scale length is not just a scatter around a narrow range of luminosities.

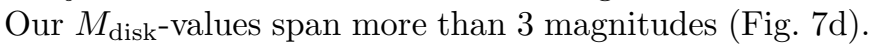
However, an upper limit in absolute disk luminosity would cause an empty upper right corner of the diagram: there exist no large disks with a high central $S B$. Our correlation has a somewhat smaller scatter than de Jong's corresponding diagram of results in the $K$ band. Moriondo et al. (1998) \& Khosroshahi et al. (2000b) observed similar relations between $S B_{\mathrm{c}}$ and $R_{\mathrm{d}}$ in their samples of NIR observations.
The absolute luminosities of the disks are also correlated with their scale lengths: large disks have a higher absolute luminosity in NIR, $M_{\text {disk }}=-15.28-$ $2.16 \log \left(R_{\mathrm{d}} / \mathrm{pc}\right) \pm 0.64$ with a correlation coefficient $r=$ 0.64 (Fig. $7 \mathrm{~d}$ ). For a similar result in the $H$ band see Pierini \& Gavazzi (1997).

\subsection{Bulge parameters}

The bulge parameters $R_{\mathrm{b}}=R_{\text {eff }}, S B_{\text {eff }}, M_{\text {bulge }}$ span a much larger dynamical range than those of the disks. They also show some more correlations with the Hubble types: the effective radii of the bulges decrease with Hubble types $(H T)$, i.e. late type spirals have smaller bulges (Fig. 8a). The regression line corresponds to $\log \left(R_{\mathrm{b}} / \mathrm{pc}\right)=3.28-$ $0.098 \mathrm{HT} \pm 0.38$ with a correlation coefficient $r=0.42$. However, similarly as for the disk components, the effective surface brightness of the bulges shows a big scatter and does practically not correlate with Hubble type (Fig. 8b).

De Jong (1996b) did not find a correlation between Hubble types and bulge effective radii. It should be 

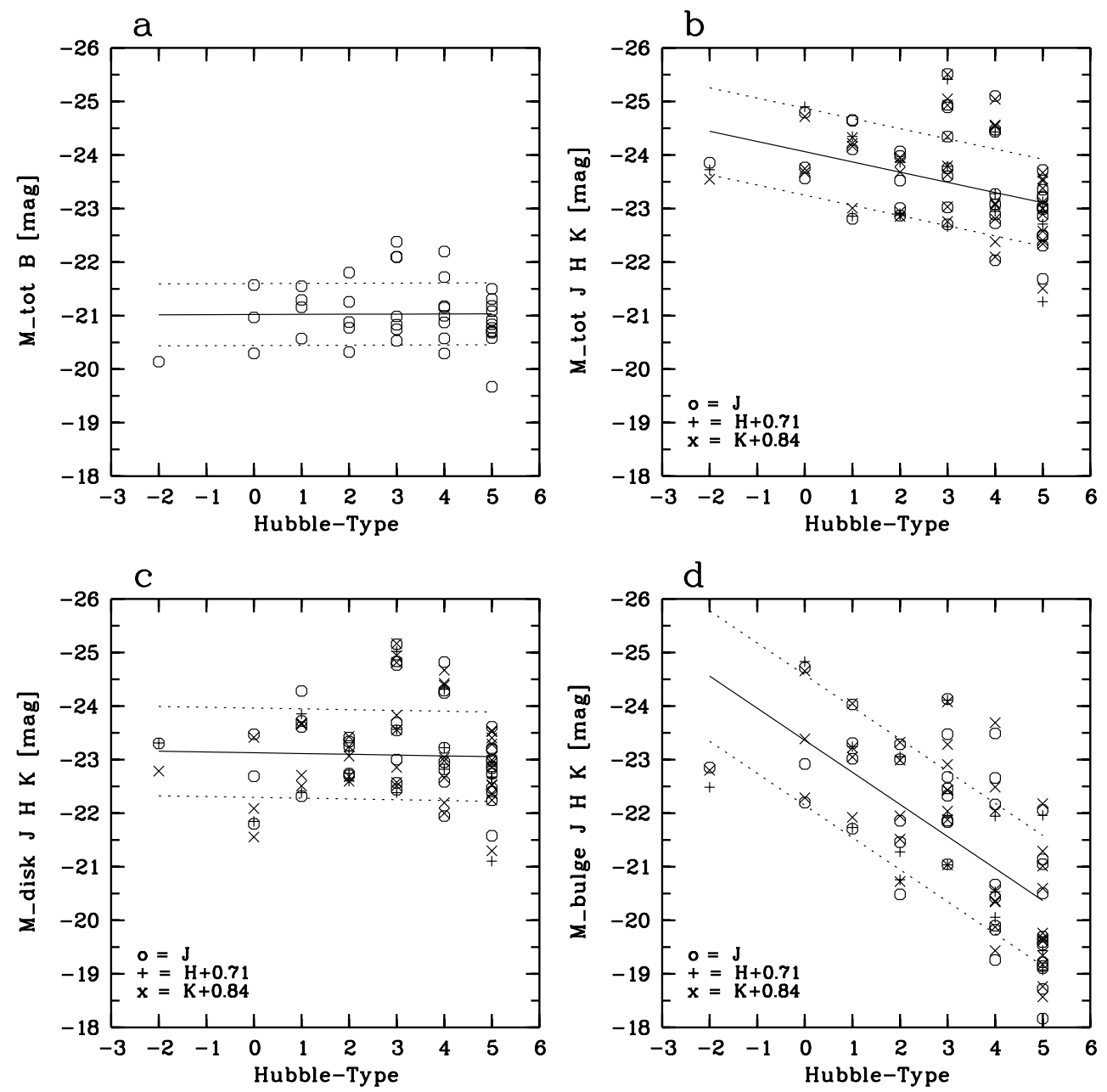

Fig. 11. (a-d) Distribution of the absolute luminosities over Hubble types (symbols and lines as in Fig. 7). Total $B$ luminosties over the Hubble types (a). The NIR absolute total luminosities of the sample galaxies decrease with Hubble types (b). While the NIR absolute luminosities of the disks only show a fairly big scatter (c), the absolute luminosities of the bulges correlate strongly with Hubble types: late spirals have fainter bulges (d)

emphasized that de Jong used exponential bulge models $(\beta=1)$ for his correlation. In contrast to that, de Jong found a correlation between effective $S B$ of the bulges and Hubble types $(H T)$, however mainly for $H T>5$, where we have no galaxies.

The correlations between the structural parameters of the bulges among themselves show similar relations as those of the disks, however more pronounced: the effective surface brightness of the bulges is dependent on the effective radius, i.e. large bulges have a lower effective surface brightness (Fig. 8c). $S B_{\text {eff }}=10.56+2.39 \log \left(R_{\mathrm{b}} / \mathrm{pc}\right) \pm$ $1.02, r=0.70$. This correlation line is again quite different from the lines of constant luminosities (dashed lines in Fig. 8c). It is the well known Kormendy relation (Kormendy 1977b). If $S B_{\text {eff }}$ is transformed to the mean $S B$ inside $R_{\text {eff }}$ a similar relation is obtained.

Andredakis et al. (1995) Moriondo et al. (1998) and Khosroshahi et al. (2000a) observed similar relations between $S B_{\text {eff }}$ and $R_{\mathrm{b}}=R_{\text {eff }}$ in their samples. These results are in contrast to de Jong (1996b) who found no correlation there. It is not clear, if this is an effect of the different choices of $\beta$.

The absolute luminosities of the bulge components are also correlated with the effective radii: large bulges have a higher absolute luminosity in NIR (Fig. 8d). The solid regression line corresponds to $M_{\text {bulge }}=-12.67-$ $2.97 \log \left(R_{\mathrm{b}} / \mathrm{pc}\right) \pm 1.04$, the correlation coefficient is $r=0.77$. Similar results were obtained by Andredakis \& Sanders (1994) for a sample of late type spirals in the $R$ band.

\subsection{Correlations of the exponent $\beta$}

The bulge exponent $\beta$ shows a statistical dependency on the Hubble type, late type spirals have a larger $\beta$, i.e. their mass distribution is less concentrated in the centre, but decreases faster outside $R_{\mathrm{b}}$ (Fig. 9a). This means that the early type spirals have bulges with flat outer profiles. The regression line through all galaxies corresponds to $\beta=0.26+0.072 H T \pm 0.24$. The correlation coefficient is only $r=0.48$, probably due to the large scatter for the 
Sc-galaxies $(H T=5)$. Andredakis et al. (1995) pointed to this correlation and tried to interpret it as an evolutionary effect (Andredakis 1998). A similar trend was also found by Khosroshahi et al. (2000b) for their sample of 26 highly inclined spirals.

The bulge exponent $\beta$ is also correlated with the bulge luminosities: the exponential bulges are fainter than the $R^{1 / 4}$-bulges (Fig. 9b). The corresponding regression is $M_{\text {bulge }}=-19.56+5.21 \log (\beta) \pm 1.18, r=0.69$.

The relation between the effective or half-light surfacebrightness $S B_{\text {eff }}$ of the bulges and the central surfacebrightness $S B_{\mathrm{c}}=S B_{\mathrm{s}}$ is given in Eq. (6). Figure 9c shows that the central $S B$ of the bulges decreases with $\beta: S B_{\mathrm{c}}=15.86+9.38 \log (\beta) \pm 1.24$, with a very high correlation coefficient $r=0.86$. This is the strongest correlation we found between the structural parameters (see also Khosroshahi et al. 2000b). The bulges with the flatter central profile and the steep outer profile ( $\beta$ large) have the lower central $S B$.

Finally there is a relation between $R_{\mathrm{b}}=R_{\mathrm{eff}}$ and $\beta$, the smaller bulges have steeper profiles outside $R_{\text {eff }}$ (Fig. 9d). The regression line corresponds to $\log \left(R_{\text {eff }}\right)=$ $2.49-1.29 \log (\beta) \pm 0.32, r=0.66$. Corresponding trends for elliptical/S0 galaxies were already observed by Caon et al. (1993) and for brightest cluster ellipses by Graham et al. (1996).

\subsection{Bulge-disk relations}

The bulge/disk ratio $(B D R)$ is correlated with Hubble type: late spirals have smaller bulge/disk ratios (Fig. 10a). Although this corresponds just to the definition of the Hubble types, the correlation is only fairly weak: $B D R=$ $0.69+0.11 H T \pm 0.24$, with a correlation coefficient $r=0.39$.

Figure 10b shows that the bulge/disk ratio decreases also with $\beta: \quad \log (B D R=0.054+1.41 \beta \pm 0.45, r=0.65$. This is a corollary to the decreasing $M_{\text {bulge }}$ for larger $\beta$-values (Fig. 9b). A similar diagram can be found in Andredakis et al. (1995) and with a weak trend also in Khosroshahi et al. (2000b).

There exist also relations between the structural parameters of the disks with regard to those of the bulges. However, these correlations are not strong. The effective $S B$ of the bulges increases with the central $S B$ of the disks (Fig. 10c): $S B_{\text {eff }}=1.11+0.94 S B_{\mathrm{c}} \pm 1.20, r=0.54$. Similarly, the effective bulge radii tend to increase with the disk scale-lengths, with a fairly large scatter (Fig. 10d): $\log \left(R_{\mathrm{b}} / \mathrm{pc}\right)=0.34+0.74 \log \left(R_{\mathrm{d}} / \mathrm{pc}\right) \pm 0.38, r=0.43$. De Jong (1996b) obtained similar weak correlations in his sample of 86 spirals for the bulge-disk parameters.

\subsection{Luminosity correlations}

The correlations of the NIR luminosities with Hubble types are of course biased by the original selection of the sample. Our original selection criterion was $B_{\text {tot }}<12$.
Figure 11a shows the $B_{\text {tot }}$-distribution of our 40 galaxies over the Hubble types, calculated using the distances from Table 1. No systematic trend is visible.

Figure 11b demonstrates how the NIR absolute total luminosities of the sample galaxies decrease with the Hubble types $(H T)$. The negative slope of the regression is easy to understand from the color differences, late spirals are bluer, respectively fainter in red. We obtain $M_{\text {tot }}=-24.06+0.19 H T \pm 0.81, r=0.39$.

Figure 11c shows that the absolute NIR luminosities of the disks are not correlated with Hubble types. This is a corollary to the fact that $R_{\mathrm{d}}$ and $S B_{\mathrm{c}}$ do not correlate with Hubble type (Figs. 7a,b). We obtain $M_{\text {disk }}=-23.13+$ $0.015 H T \pm 0.83, r=0.32$. This is in contrast to de Jong (1996b) who found decreasing disk luminosities with the Hubble types, however practically only for Hubble types between 4 and 10. Our sample ends at $H T=5$.

In contrast to that, there exists a strong correlation between the absolute NIR luminosities of the bulges with the Hubble types (Fig. 11d), late spirals have fainter bulges. We obtain $M_{\text {bulge }}=-23.36+0.60 H T \pm 1.22, r=0.67$ It is practically only the decreasing contribution of the bulges which produces the decreasing bulge/disk ratio with Hubble types (Fig. 10a). Concerning the total luminosities, the fairly strong dependency of the bulge luminosities on the Hubble types is diluted by the nearly non-dependency of the disks, resulting in the weaker dependency of $M_{\text {tot }}$ in Fig. 11b.

\section{Discussions}

\subsection{Variable bulge slope exponent $\beta$}

The bulge exponent $\beta=1 / n$ is a quantity which defines the steepness or the mass concentration of the bulges. For a given galaxy, the choice of $\beta$ has a strong influence on the size of the effective radius resulting from a fit. In that respect $R_{\text {eff }}$ and $\beta$ are not totally independent. However, a fit with a given fixed $\beta$ does not lead to an optimal model as we have demonstrated in Sect. 4.2. The strong influence of the choice of a fixed $\beta$ especially on the bulge parameters was also discussed in Sect. 5.3. It is an important result of this study, that $\beta$ is better treated as an additional variable than as a fixed exponent. This result is supported by the studies of Andredakis et al. (1995); Khosroshahi et al. (2000a,b), and also by the 1-dim results of Seigar \& James (1998).

The bulge exponent $\beta$ shows a clear statistical dependency on the Hubble type: late spirals have steeper bulges (outside $R_{\text {eff }}$ ), however with a large scatter for the Scgalaxies $(H T=5$, see Fig. 9a). This correlation, also found by Andredakis et al. (1995) and Khosroshahi et al. (2000b), might be explained by the gravitational influence of a disk formed later in the history of the galaxy. Andredakis (1998) studied via $n$-body calculations the influence of a disk of increasing mass on a bulge. The disk changed an originally $\beta=0.25$ of the bulge to higher values. 
These results on the $\beta$ behaviour would support a formation scenario of spiral galaxies, where during a first monolithic collapse the bulge with $\beta \approx 0.25$ is formed, similar to an elliptical galaxy. The disk would then be formed in a later stage and would change the $\beta$ of the bulge subsequently to higher values, according to the final mass of the disk. Our observations of an decreasing bulge/disk ratio with $\beta$ fits well to this scenario (Fig. 10a).

\subsection{Exponential bulges?}

There are some results in the literature which favour scenarios for galaxy evolution where the disk was formed first and the bulge later by secular evolution. Courteau et al. (1996) used exponential profiles for the disks as well as for the bulges. They found $R_{\mathrm{b}} / R_{\mathrm{d}}=0.08 \pm 0.05$ for a large sample of $\approx 300$ spirals, uncorrelated with the Hubble types (1-dim fit method).

Andredakis \& Sanders (1994) studied 34 late type spirals in the $R$ band with two different bulge models: $\beta=1$ and $\beta=0.25$. They found that the relations $M_{\text {bulge }}$ vs. $S B_{\text {eff }}$ and $M_{\text {disk }}$ vs. $M_{\text {bulge }}$ are tighter for the exponential bulge $(\beta=1)$ than for the de Vaucouleurs bulge $(\beta=0.25)$. They stated that the exponential bulge fit is superior in the statistical aspects of the fitting procedure, in the sense that various goodness-of-fit indicators are better.

These results would support the following scenario of galaxy evolution: i) formation of an exponential disk, ii) secular dynamical evolution of the disk leading to a bar instability, iii) viscous transport of disk material into the central region, iii) formation of a bulge with a size dependent on disk size.

The results with the exponential bulge profiles are not a contradiction to our preference for a variable $\beta$ since Andredakis \& Sanders (1994) considered only Sb and Sc galaxies, i.e. late types where an approximation with a fixed large $\beta=1$ is not too bad. However, we gave strong evidence that $\beta$ increases from 0.25 to 1 over the Hubble types. We also found that $R_{\mathrm{b}} / R_{\mathrm{d}}$ is not constant but decreases with Hubble types. Generally, our 2-dim studies, as well as those of other authors (Andredakis et al. 1995; Seigar \& James 1998) demonstrated that exponential profiles are not realistic for all Hubble types.

This means that our results again favour a scenario where the bulge was formed first. However, this does not exclude a secular later formation of bulges. Probably both scenarios may take place during galaxy evolution.

It is clear that morphological studies alone do not provide sufficient information to distinguish between the various formation scenarios. Systematical studies of the kinematics and the stellar populations in spiral galaxies are additionally required.

\subsection{Fundamental plane for bulges and disks}

Some of the correlations described in the last section are especially interesting: i) The relation $S B_{\text {eff }}$ vs. $\log \left(R_{\text {eff }}\right)$ shows that large bulges have a lower effective surface

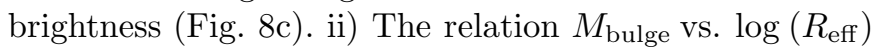
shows that large bulges have a higher absolute luminosity in NIR (Fig. 8d).

These correlations are part of the global scaling relations for the structural parameters of elliptical galaxies and bulges which populate a narrow strip in the fundamental plane (Djorgovski \& Davis 1987; Faber et al. 1987; Bender et al. 1992, 1993).

It was shown in the last section that the disks show very similar relations, although not as pronounced as the bulges (Figs. 7c,d). In order to compare the disks directly with the bulges we calculate the effective or half-light radii $R_{\text {eff }}$ and $S B_{\text {eff }}$ for the disks using Eq. (6). In Fig. 12a we plot these quantities for the disks (crosses) as well as those for the bulges (circles). Obviously both components follow the same relation $S B_{\text {eff }}=11.07+2.24 \log \left(R_{\text {eff }} / \mathrm{pc}\right) \pm 0.84$, the correlation coefficient is $r=0.82$. Similarly we obtain in Fig. $12 \mathrm{~b} M_{\text {bulge,disk }}=-14.68-2.25 \log \left(R_{\text {eff }} / \mathrm{pc}\right) \pm 0.91$, $r=0.80$. The disks populate the regions with the larger $R_{\text {Coneff-values, the lower } S B \text {-values and the higher abso- }}$ lute luminosities, i.e. the same region where the bright and large bulges can be fiound. A similar location of elliptical and of spiral galaxies in the fundamental plane was already found by Burstein et al. (1993), called cosmic metaplane. Burstein et al. obtained the parameters of the spirals directly from the global quantities as given in the RC3 (de Vaucouleurs et al. 1991). Here we could show the common distribution of disks and bulges in the fundamental plane if the corresponding spiral galaxies are carefully decomposed. Disks and bulges follow the same relations between their characteristic size, surface brightness and luminosity (mass). It is not surprising that the bulges and disks of spiral galaxies populate similar regions in the $S B_{\text {eff }}$ vs. $\log \left(R_{\text {eff }} / \mathrm{pc}\right)$ plane: all bulges and disks are virialized systems and have similar mass-to-light ratios (Bender et al. 1993). The dissipation of the disks occurred mainly in $z$-direction and is not visible on our face-on analysis.

\subsection{Photometric plane for bulges}

Khosroshahi et al. (2000a) found that the structural parameters of elliptical galaxies and of bulges form a photometric plane in a space built up by $\beta=$ $1 / n, \log \left(R_{\text {eff }} / \mathrm{pc}\right), S B_{\mathrm{c}}$. Here $S B_{\mathrm{c}}$ is the central surface brightness of the bulges. Using Eqs. (5), (6) it can be obtained from the effective $S B$ by

$$
S B_{\mathrm{c}}=S B_{\mathrm{eff}}-1.0857 b_{\mathrm{e}}=S B_{\mathrm{eff}}-2.163 / \beta+0.354 .
$$

Khosroshahi et al. (2000b) describe the photometric plane for the bulges by the linear combination of parameters

$\log (1 / n)=0.130 \log \left(R_{\text {eff }} / \mathrm{pc}\right)-0.073 S B_{\mathrm{c}}+1.21 \pm 0.11$. 

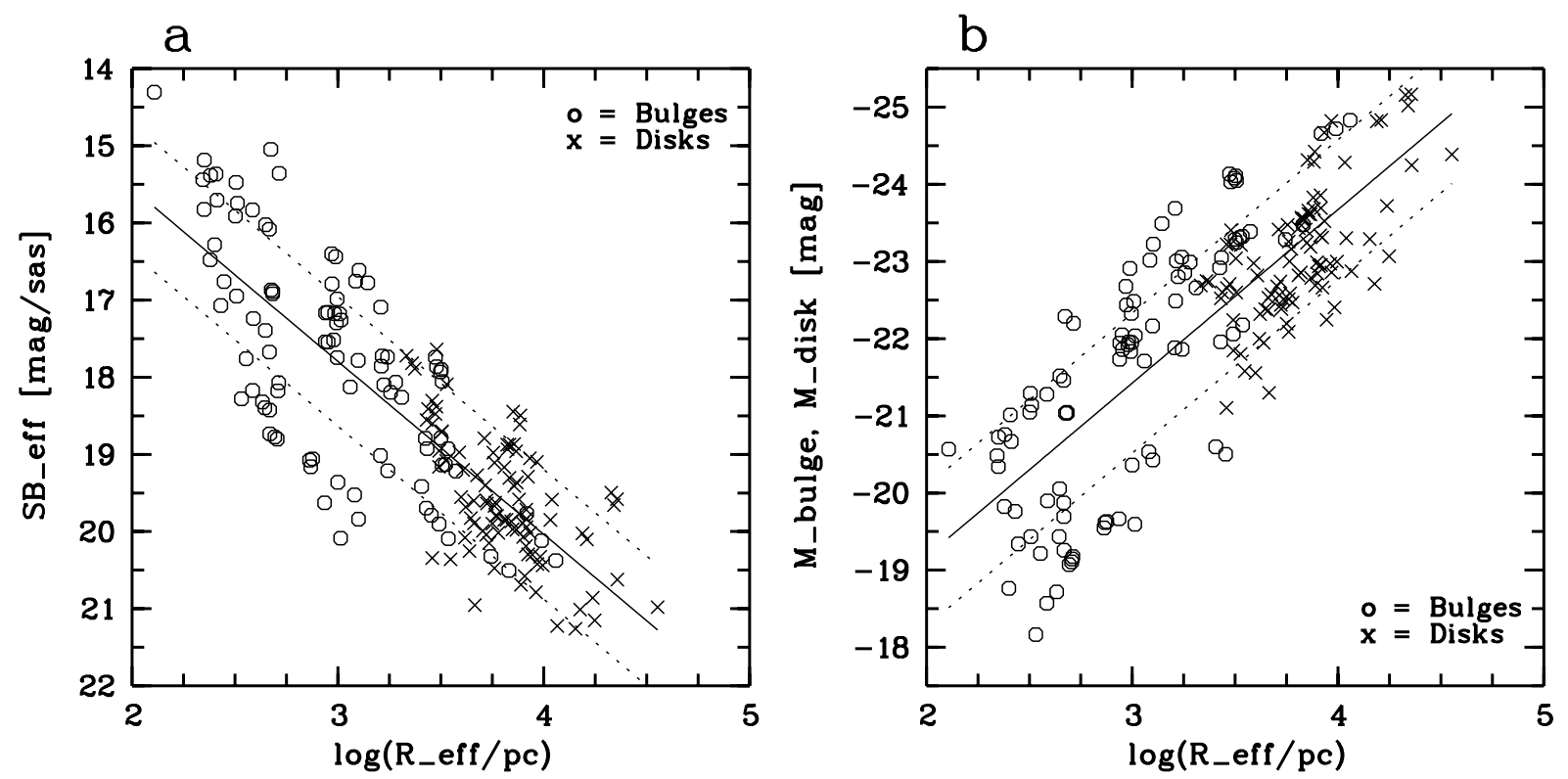

Fig. 12. (a and b) Disks and bulges follow the same relations in the fundamental plane $(\times=$ disks, $\circ=$ bulges $)$, namely a correlation between $S B_{\text {eff }}$ and $\log \left(R_{\text {eff }} / \mathrm{pc}\right)$ (a) and a correlation between $M_{\text {disk, bulge }} \operatorname{and} \log \left(R_{\text {eff }} / \mathrm{pc}\right)(\mathbf{b})$

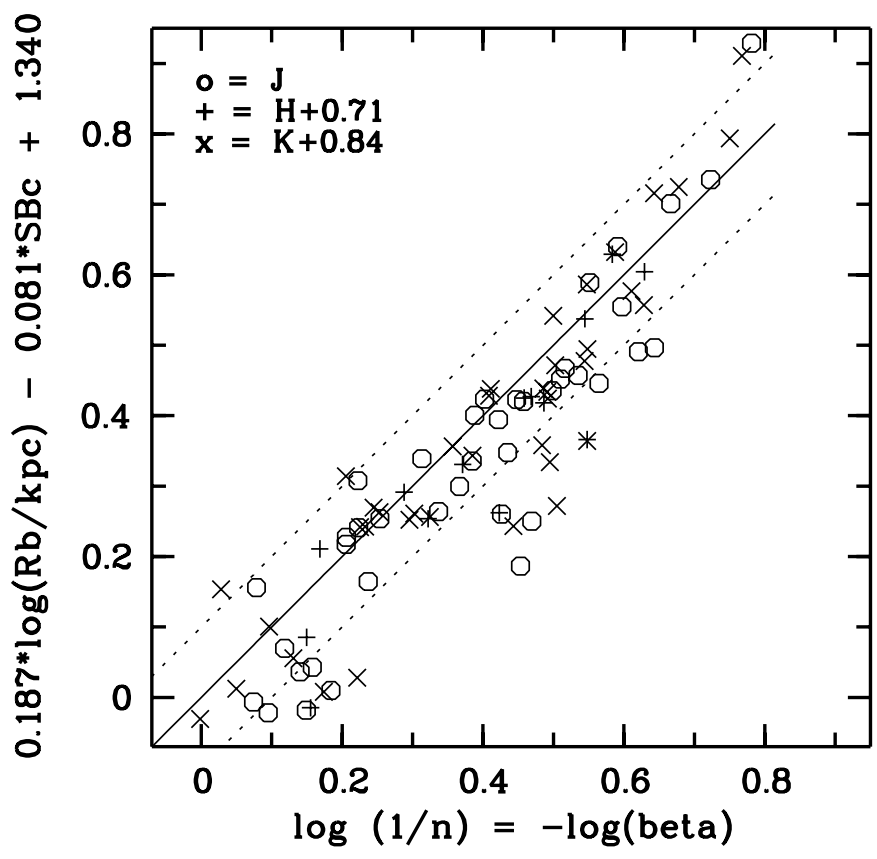

Fig. 13. The linear combination of $\log \left(R_{\mathrm{b}}\right)$ and the central surface brightness $S B_{\text {c }}$ correlate well with $\log (\beta)$. The diagram shows an edge-on view of the photometric plane in the $\log (\beta), \log \left(R_{\text {eff }} /\right.$ pc $), S B_{\mathrm{c}}-$ space

Performing a bivariate regression in this parameter space we obtained from our sample the similar relation

$\log (1 / n)=0.187 \log \left(R_{\text {eff }} / \mathrm{pc}\right)-0.081 S B_{\mathrm{c}}+1.34 \pm 0.10$.

Figure 13 shows the structural parameters of our sample in a corresponding diagram, with a correlation coefficient $r=0.91$. The differences between our coefficients in the relation above and those of Khosroshahi et al. are within the error limits which they gave, although the samples are of somewhat different character: our sample is dominated by late type spirals $(H T=5)$ and low inclination objects, while their sample contains more early types and high inclination objects. Obviously the bulges of all spirals are placed on the same photometric plane. This is a further hint to a common formation scenario.

This correlation in a photometric plane works only if one uses the central surface brightness $S B_{\mathrm{c}}$ of the bulges. A corresponding narrow photometric plane does not occur, if one replaces the central surface brightness $S B_{\mathrm{c}}$ by the effective surface brightness $S B_{\text {eff }}$ or by the mean surface brightness inside $R_{\text {eff }}$. Vice versa, while $S B_{\text {eff }}$ vs. $\log \left(R_{\text {eff }} / \mathrm{pc}\right)$ form a fairly tight relation (Fig. 8c), this is not the case for $S B_{\mathrm{c}}$ vs. $\log \left(R_{\text {eff }} / \mathrm{pc}\right)$.

The astrophysical cosequences of the tight relation between $\log (\beta)$ and the linear combination of $\log \left(R_{\mathrm{eff}} / \mathrm{pc}\right)$ and $S B_{\mathrm{c}}$ is not yet clear. Khosroshahi et al. (2000a,b) showed that it is not just an internal correlation between the chosen structural parameters.

\section{Conclusions}

We have presented images in $J, H$, and $K$ of a sample of bright spiral galaxies with low inclination and of different Hubble types. These allow to study the distribution of the stellar mass distribution in an optimal manner.

A two-dimensional method to fit the surface-brightness distribution was developed which describes the disks with an exponential function and the bulges with a generalized $\exp \left(-R^{\beta}\right)$ function. It was demonstrated that a variable mass-concentration exponent $\beta$ is necessary to give a good description of the bulge profiles. A fit with a fixed exponent does not give an adequate result. A simultaneous fit of these 2-dim functions to the whole observed pixel image yielded an optimal bulge-disk deconvolution together with 
the characteristic structural parameters. Main sources of possible errors were internal asymmetries in the galaxies, the limited field of view, the exact determination of the sky background, and a precise description of the seeing PSF.

The structural parameters of the bulges and disks were correlated with other global parameters of the galaxies (Hubble types, luminosities) and among themselves, leading to a number of interesting relations. The main results were that bright disks have larger scale lengths, bulges show the same dependency. Bulges and disks populate the same strip in the fundamental plane. Bright bulges are also less concentrated, the exponent $\beta$ is smaller.

There exists a relation between the exponent $\beta$ and the bulge/disk ratio: early type spirals with strong bulges have $\beta \approx 0.25$ similar to elliptical galaxies, whereas the late type spirals with tiny bulges have exponential profiles $(\beta \approx 1)$. This relation supports an evolutionary scenario proposed by Andredakis (1998), where the galaxy formation started with an $R^{1 / 4}$ bulge while the disk was formed later. According to the increasing mass fraction of the disk the bulge adjusts to a profile which is less concentrated in the center but decreases faster outside (larger $\beta$ ).

The structural parameters of the bulges form a photometric plane in the $\beta=1 / n, \log \left(R_{\text {eff }} / \mathrm{pc}\right), S B_{\mathrm{c}}$ space. The existence of this narrow plane is a further hint to a common formation scenario of all types of spiral galaxies.

Acknowledgements. This project was supported by the Sonderforschungsbereich 328 of the Deutsche Forschungsgemeinschft (DFG). We are grateful to the Calar Alto team for the effective support during the observations. We acknowledge the use of the SIMBAD database (CNRS data centre, Strasbourg).

\section{References}

Afanasiev, V. L., \& Sil'chenko, O. K. 1999, AJ, 117, 1725

Andredakis, Y. C. 1998, MNRAS, 295, 725

Andredakis, Y. C., Peletier, R. F., \& Balcells, M. 1995, MNRAS, 275,874

Andredakis, Y. C., \& Sanders, R. H. 1994, MNRAS, 267, 283

Baggett, W. E., Baggett, S. M., \& Anderson, K. S. J. 1998, AJ, 116, 1626

Bender, R., Burstein, D., \& Faber, S. M. 1992, ApJ, 399, 462

Bender, R., Burstein, D., \& Faber, S. M. 1993, ApJ, 411, 153

Bender, R., Burstein, D., \& Faber, S. M. 1997, in Galaxy scaling relations, ed. L. N. da Costa, \& A. Renzini (Springer, Berlin), 95

Bender, R., Döbereiner, S., \& Möllenhoff, C. 1988, A\&AS, 74, 385

Bender, R., \& Möllenhoff, C. 1987, A\&A, 177, 71

Bender, R., Surma, P., Döbereiner, S., Möllenhoff, C., \& Madejsky, R. 1989, A\&A, 217, 35

Bevington, P. R. 1969, Data reduction and error analysis (McGraw-Hill, New York)

Boer, B., Schulz, H., \& Keel, W. C. 1992, A\&A, 260, 67

Böker, T., Calzetti, D., Sparks, W., et al. 1999, ApJS, 124, 95

Boroson, T. 1981, ApJS, 46, 177

Bottinelli, L., Gouguenheim, L., Paturel, G., \& de Vaucouleurs, G. 1984, A\&AS, 56, 381
Bottinelli, L., Gouguenheim, L., Paturel, G., \& de Vaucouleurs, G. 1985a, A\&AS, 59, 43

Bottinelli, L., Gouguenheim, L., Paturel, G., \& de Vaucouleurs, G. 1985b, ApJS, 59, 293

Braun, R., Walterbos, R. A. M., Kennicutt, R. C., \& Tacconi, L. J. 1994, ApJ, 420, 558

Broeils, A. H., \& Courteau, S. 1997, in Dark and Visible Matter in Galaxies, ed. M. Persic, \& P. Salucci, ASP Conf. Ser., 117,74

Burstein, D., Bender, R., Faber, S. M., \& Nolthenius, R. 1997, AJ, 114, 1365

Byun, Y. I. 1992, Ph.D. Thesis, Australian National University Byun, Y. I., \& Freeman, K. 1995, ApJ, 448, 563

Caon, N., Capaccioli, M., \& D'Onofrio, M. 1993, MNRAS, 265, 1013

Carollo, C. M., Stiavelli, M., \& Mack, J. 1998, AJ, 116, 68

Carollo, C. M., Ferguson, H. C., \& Wyse, R. F. G. 1999, The Formation of Galactic Bulges (Cambridge Univ. Press)

Ciri, R., Bettoni, D., \& Galletta, G. 1995, Nature, 375, 661

Colina, L., Garcia Vargas, M. L., Mas-Hesse, J. M., Alberdi, A., \& Krabbe, A. 1997, ApJ, 484, L41

Colina, L., \& Arribas, S. 1999, ApJ, 514, 637

Corradi, R. L. M., Boulesteix, J., Bosma, A., Capaccioli, M., \& Marcelin, M. 1991, A\&A, 244, 27

Corsini, E. M., Pizzella, A., Sarzi, M., et al. 1999, A\&A, 342, 671

Courteau, S. 1996, ApJS, 103, 363

Courteau, S., de Jong, R. S., \& Broeils, A. H. 1996, ApJ, 457, L73

de Jong, R. S. 1996a, A\&AS, 118, 557

de Jong, R. S. 1996b, A\&A, 313, 45

de Jong, R. S. 1996c, A\&A, 313, 377

de Vaucouleurs, G. 1953, MNRAS, 113, 134

de Vaucouleurs, G., de Vaucouleurs, A., Corwin, H. G., et al. 1991, Third Reference Catalogue of Bright Galaxies (RC3) (Springer, New York, Heidelberg)

Djorgovski, S., \& Davis, M. 1987, ApJ, 313, 59

Elias, J. H., Frogel, J. A., Matthews, K., \& Neugebauer, G. 1982, AJ, 87, 1029

Emsellem, E., Monnet, G., Bacon, R., \& Nieto, J. L. 1994, A\&A, 285, 739

Erwin, P., Sparke, L. S., \& Gallagher, J. S. 1996, AAS, 188, 1002

Eskridge, P. B., Frogel, J. A., Pogge, R. W., et al. 2000, AJ, 119,536

Faber, S. M., Dressler, A., Davies, R. L., et al. 1987, in Nearly normal galaxies, ed. S. M. Faber (Springer, Heidelberg), 175

Freeman, K. C. 1970, ApJ, 160, 811

Federspiel, M., Tammann, G. A., \& Sandage, A. 1998, ApJ, 495, 115

Ferrarese, L., Mould, J. R., Kennycutt, R. C., et al. 2000, ApJ, 529,745

Frogel, J. A., Quillen, A. C., \& Pogge, R. W. 1996, in New Extragalactic Perspectives in New South Africa, ed. D. Block (Kluwer, Dordrecht), 65

Gibson, B. K., Hughes, S. M. G., Stetson, P. B., et al. 1999, ApJ, 512, 48

Gibson, B. K., Stetson, P. B., Freedman, W. L., et al. 2000, ApJ, 529, 732

Graham, A., Lauer, T. R., Colless, M., \& Postman, M. 1996, ApJ, 465, 534

Grosbøl, P. J. 1985, A\&AS, 60, 261 
Héraudeau, P., \& Simien, F. 1996, A\&AS, 118, 111

Héraudeau, P., Simien, F., \& Mamon, G. A. 1996, A\&AS, 117, 417

Héraudeau, P., \& Simien, F. 1997, A\&A, 326, 897

Herbst, T. M., Beckwith, S. V. W., Birk, C., et al. 1993, in Infrared detectors and instrumentation, Proc. SPIE, 1946, Bellingham, 605

Huchtmeier, W. K., \& Richter, O. G. 1982, A\&A, 109, 331

Jogee, S., Kenney, J. D. P., \& Smith, B. J. 1998, ApJ, 185, L85

Jogee, S., Kenney, J. D. P., \& Smith, B. J. 1999, ApJ, 526, 665

Jungwiert, B., Combes, F., \& Axon, D. J. 1997, A\&AS, 125, 479

Keel, W. C. 1983, ApJ, 268, 632

Kelson, D. D., Illingworth, G. D., Saha, A., et al. 1999, ApJ, 514,614

Kent, S. 1985, ApJS, 59, 115

Knapen, J. H., \& van der Kruit, P. C. 1991, A\&A, 248, 57

Khosroshahi, H. G., Wadadekar, Y., Kembhavi, A., \& Mobasher, B. 2000a, ApJ, 531, L103

Khosroshahi, H. G., Wadadekar, Y., \& Kembhavi, A. 2000b, ApJ, 533, 162

Kodaira, K., Watanabe, M., \& Okamura, S. 1986, ApJS, 62, 703

Kodaira, K., Okamura, S., \& Ichikawa, S. 1990, Photometric atlas of northern bright galaxies (Univ. of Tokyo Press)

Kormendy, J. 1977a, ApJ, 217, 406

Kormendy, J. 1977b, ApJ, 218, 333

Laine, S., Knapen, J. H., Pérez-Ramirez, D., Doyon, R., \& Nadeau, D. 1999, MNRAS, 302, L33

Lu, N. Y. 1998, ApJ, 506, 673

Macri, L. M., Huchra, J. P., Stetson, P. B., et al. 1999, ApJ, 521,155

Martin, P. 1995, AJ, 109, 2428

Möllenhoff, C. 1999, in The Formation of Bulges, ed. C. M. Carollo, H. C. Ferguson, \& R. F. G. Wyse (Cambridge Univ. Press), 157

Möllenhoff, C. 2001, in preparation

Möllenhoff, C., Appenzeller, I., Gässler, W., et al. 1999, A\&A, 352, L5

Monnet, G., Bacon, R., \& Emsellem, E. 1992, A\&A, 253, 366

Moriondo, G., Giavonardi, C., \& Hunt, L. K. 1998, A\&AS, 130, 81

Natali, G., Pedechini, F., \& Righini, M. 1992, A\&A, 256, 79

Okamura, S. 1988, PASP, 100, 524

Peletier, R. F., \& Balcells, M. 1996, AJ, 111, 2238

Phillips, A. C., Illingworth, G. D., MacKenty, J. W., \& Franx, M. 1996, AJ, 111, 1566

Pierini, D, \& Gavazzi, G. 1997, in Galaxy Scaling Relations, ed. L. N. da Costa, \& A. Renzini (Springer-Verlag), 381

Pompei, E., \& Natali, G. 1997, A\&AS, 124, 129

Press, W. H., Flannery, B. P., Teukolsky, S. A., \& Vetterling, W. T. 1989, Numerical Recipes (Cambridge Univ. Press)

Prieto, M., Beckman, J. E., Cepa, J., \& Varela, A. M. 1992, A\&A, 257, 85

Rubin, V. C. 1994, AJ, 107, 173

Rubin, V. C., Kenney, J. D. P., \& Young, J. S. 1997, AJ, 113, 1250

Sakai, S., Mould, J. R., Hughes, S. M. G., et al. 2000, ApJ, 529, 698

Sandage, A., \& Bedke, J. 1994, The Carnegie Atlas of Galaxies, Canegie, Inst., Washington

Sandage, A., \& Tammann, G. A. 1981, A revised ShapleyAmes catalog of bright galaxies, Carnegie Institution, Washington

Sérsic, J. L. 1968, Atlas de galaxias australes, Observatorio Astronómico de Córdoba

Schneider, S. E. 1989, ApJ, 343, 94

Schombert, J. M., \& Bothun, G. D. 1987, AJ, 93, 60

Scorza, C., \& Bender, R. 1990, A\&A, 235, 49

Scorza, C., \& Bender, R. 1995, IAU Symp., No. 171 (Kluwer, Dordrecht)

Seigar, M. S., \& James, P. A. 1998, MNRAS, 299, 672

Shanks, T. 1997, MNRAS, 290, L77

Sharina, M. E., Karachentsev, I. D., \& Tikhonov, N. A. 1996, A\&AS, 119, 499

Sil'chenko, O. K. 1999, AJ, 117, 2725

Simien, F. 1989, in The world of galaxies, ed. H. G. Corwin, \& L. Bottinelli, 293

Simien, F., \& de Vaucouleurs, G. 1986, ApJ, 302, 564

Simien, F., \& Prugniel, Ph. 1997, A\&AS, 126, 519

Smith, B. J. 1991, ApJ, 378, 39

Storchi-Bergmann, T., Wilson, A. S., \& Baldwin, J. A. 1996, ApJ, 460, 252

Tanvir, N. R., Ferguson, H. C., \& Shanks, T. 1999, MNRAS, 310,175

Tully, B. R. 1988, Nearby Galaxies Catalog, Cambridge

Turner, A., Ferrarese, L., Saha, A., et al. 1998, ApJ, 505, 207

Varela, A. M., Munõz-Tunõn, C., \& Simmoneau, E. 1996, A\&A, 306, 381

Wadadekar, Y., Robbason, B., \& Kembhavi, A. 1999, AJ, 117, 1219

Walterbos, R. A. M., Braun, R., \& Kennicutt, R. C. 1994, AJ, 107,184

Yoshida, M., Taniguchi, Y., \& Murayama, T. 1999, AJ, 117, 158 